\title{
Achievement of a low-loss 1-MW beam operation in the 3-GeV rapid cycling synchrotron of the Japan Proton Accelerator Research Complex
}

\author{
H. Hotchi, ${ }^{*}$ H. Harada, N. Hayashi, S. Kato, M. Kinsho, K. Okabe, P. K. Saha, Y. Shobuda, \\ F. Tamura, N. Tani, Y. Watanabe, K. Yamamoto, M. Yamamoto, and M. Yoshimoto \\ Accelerator division, J-PARC center, Japan Atomic Energy Agency (JAEA), Tokai, \\ Naka, Ibaraki 319-1195 Japan
}

(Received 7 March 2017; published 19 June 2017)

\begin{abstract}
The 3-GeV rapid cycling synchrotron (RCS) of the Japan Proton Accelerator Research Complex (J-PARC) is now in the final beam commissioning phase, aiming for a design output beam power of $1 \mathrm{MW}$. With a series of injector linac upgrades in 2013 and 2014, RCS developed a high-intensity beam test, and launched 1-MW beam tuning in October 2014. The most important issues in realizing such a high-power continuous beam operation are to control and minimize beam loss for maintaining machine activations within permissible levels. In RCS, numerical simulation was successfully utilized along with experimental approaches to isolate the mechanism of beam loss and find its solution. By iteratively performing actual beam experiments and numerical simulations, and also by several hardware improvements, we have recently established a 1-MW beam operation with very low fractional beam loss of a couple of $10^{-3}$. In this paper, our recent efforts toward realizing such a low-loss high-intensity beam acceleration are presented as a follow-up of our previous article, H. Hotchi et al. Phys. Rev. ST Accel. Beams 12, 040402 (2009), in which the initial beam commissioning status of RCS has been reported.
\end{abstract}

DOI: 10.1103/PhysRevAccelBeams.20.060402

\section{INTRODUCTION}

The Japan Proton Accelerator Research Complex (J-PARC) [1] is a multipurpose high-power proton accelerator facility comprising a 400-MeV linac, a 3-GeV rapid cycling synchrotron (RCS), a 50-GeV main ring synchrotron (MR), and three experimental facilities - a materials and life science experimental facility (MLF), a hadron experimental facility (HD), and a neutrino experimental facility (NU). In this chain of accelerators, RCS has two functions, namely, as a proton driver for producing pulsed muons and neutrons at MLF and as an injector to MR, a schematic view of which is shown in Fig. 1.

The design output beam power of RCS is $1 \mathrm{MW}$, which, when commissioned, would be the highest class in the world. Figure 2 shows the history of the RCS output beam power. RCS was beam commissioned in October 2007 and made available for the user program in December 2008 with an output beam power of $4 \mathrm{~kW}[2,3]$. Since then, the RCS beam power ramp-up has steadily proceeded as per progressions in beam tuning and hardware improvements. The routine output beam power for the user program has been increased to $500 \mathrm{~kW}$ to date.

*hotchi.hideaki@jaea.go.jp

Published by the American Physical Society under the terms of the Creative Commons Attribution 4.0 International license. Further distribution of this work must maintain attribution to the author(s) and the published article's title, journal citation, and DOI.
Recently, injector linac upgrades have been completed, by which the injection energy was upgraded from $181 \mathrm{MeV}$ to the design value of $400 \mathrm{MeV}$ in 2013, and then the injection peak current was increased from $30 \mathrm{~mA}$ to the design value of $50 \mathrm{~mA}$ in 2014. By these series of injector linac upgrades, all hardware parameters to realize the design performance of RCS were in place. From then on, a fullscale high-intensity beam test has been developed between operational duties for users. As indicated by the red bars in Fig. 2, we first conducted high-intensity beam tests of up to $600 \mathrm{~kW}$ for both injection energies of $181 \mathrm{MeV}$ and $400 \mathrm{MeV}$ before and after the injection energy upgrade,

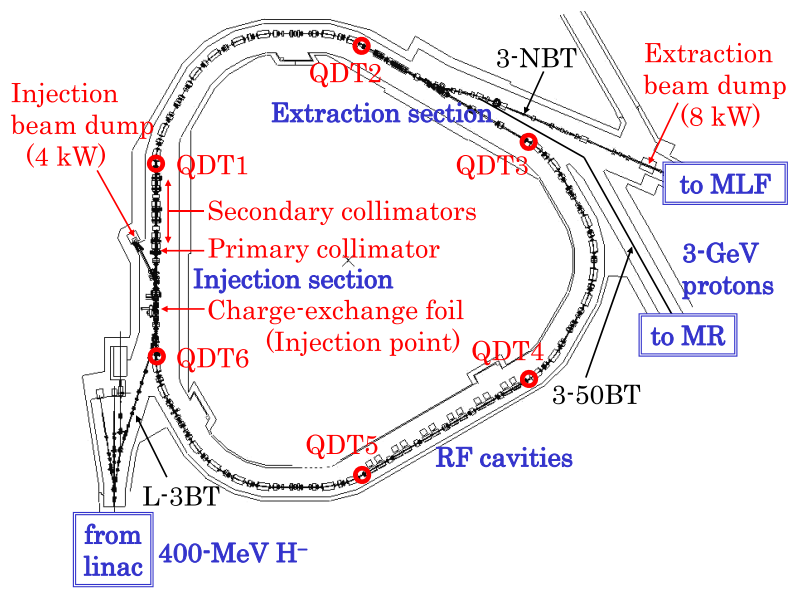

FIG. 1. Schematic view of the J-PARC 3-GeV RCS. 


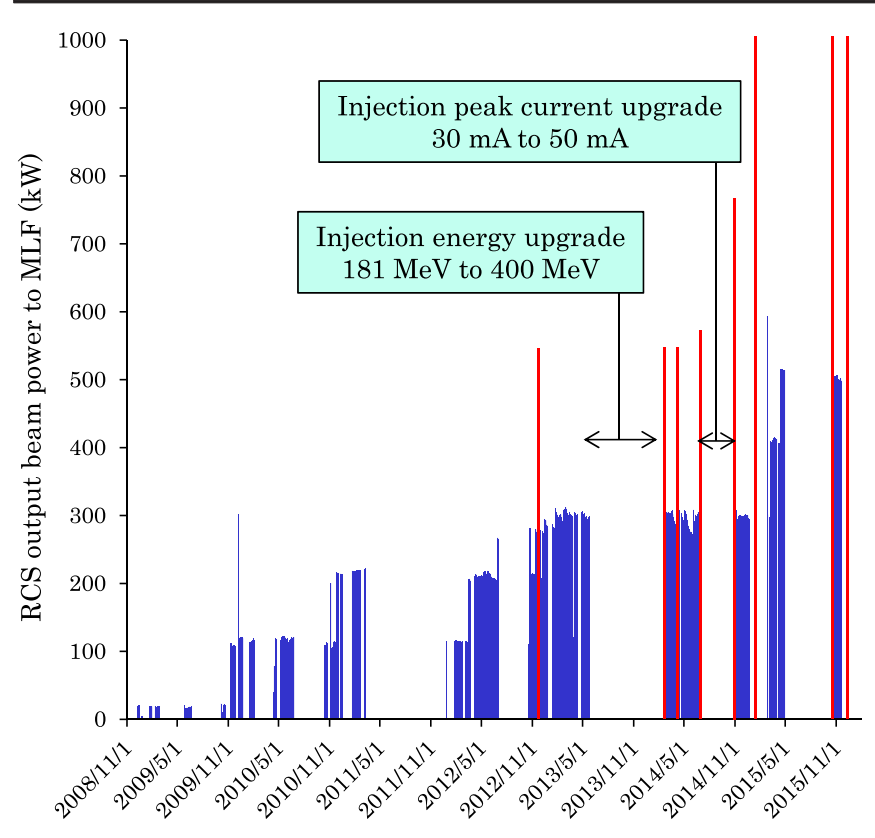

FIG. 2. Beam power ramp-up history of RCS since the start-up of user programs in December 2008, where the blue bars correspond to the beam power in the routine user operation at $25 \mathrm{~Hz}$, while the red ones show the equivalent beam power achieved by the beam-on-demand single-shot mode in the beam test.

and then launched a 1-MW beam test in October 2014 right after the injection beam current upgrade.

The most important issues in realizing such a MW-class high-power beam operation are controlling and minimizing beam loss to maintain machine activations within permissible levels. In high-power machines such as RCS, there exist many factors causing beam loss, such as lattice imperfections, space charge, image charge, beam instability, and foil scattering. Besides, beam loss generally occurs through a complex mechanism involving several factors. To clarify such a beam loss mechanism and realize its cure, conducting numerical simulation as well as experiments are essential. In RCS, numerical simulation well reproduces empirical data, and now plays a vital role in solving beam loss issues in combination with actual beam experiments; various ideas for beam loss mitigation were proposed with the help of the numerical simulations, and verified by experiments. As a result of such continuous efforts including several hardware improvements, we have recently accomplished a 1-MW beam acceleration with considerably low fractional beam loss of several $10^{-3}$.

This paper presents recent progresses of RCS beam commissioning in chronological order with particular emphasis on our approaches to beam loss issues that we faced in a series of high-intensity beam tests of up to $1 \mathrm{MW}$, and also explains how well the numerical simulation functioned for realizing such a low-loss 1-MW beam operation by way of example.

\section{OUTLINE OF J-PARC 3-GeV RCS}

The RCS design parameters are summarized in Table I. As shown in Fig. 1, a 400-MeV $H^{-}$beam from the linac is delivered via the linac-to-3-GeV beam transport line (L-3BT) to the RCS injection point, where it is multiturn charge-exchange injected through a $340-\mu \mathrm{g} / \mathrm{cm}^{2}$-thick hybrid-type boron-mixed carbon-stripping foil [4] over a period of $0.5 \mathrm{~ms}$. In order to avoid longitudinal beam loss during injection, the $H^{-}$linac beam is equipped with a chopped bunch structure in synchronization with the ring rf frequency at the time of injection, where the chopper beam-on duty factor is typically set to $53 \%-60 \%$. RCS accelerates the injected protons up to $3 \mathrm{GeV}$ with a repetition rate of $25 \mathrm{~Hz}$. Most of the $3-\mathrm{GeV}$ beam pulses from RCS are transported via the 3-GeV-toneutron-target beam transport line (3-NBT) to MLF, while only four pulses every several seconds are transported via the $3-\mathrm{GeV}$-to-50-GeV beam transport line (3-50BT) to MR.

RCS has a three-fold symmetric lattice over its circumference of $348.333 \mathrm{~m}$. Each super-period consists of two 3 -DOFO arc modules and one 3-DOFO long straight insertion, where DOFO stands for a defocusing-focusing periodic cell. Each arc module has a missing-bend cell, where the horizontal dispersion has the maximum $(6 \mathrm{~m})$. Such a lattice structure gives a high transition energy

TABLE I. RCS design parameters.

\begin{tabular}{lc}
\hline \hline Circumference & $348.333 \mathrm{~m}$ \\
Superperiodicity & 3 \\
Injected particles & $H^{-}$ \\
Injection energy & $400 \mathrm{MeV}$ \\
Injection period & $0.5 \mathrm{~ms}$ \\
Injection turns & 307 \\
Injection peak current & $50 \mathrm{~mA}$ \\
Chopper beam-on duty factor of the & $53.3 \%$ \\
$\quad$ injection beam & \\
Unnormalized transverse emittance of the & $4 \pi \mathrm{mm} \mathrm{mrad}$ \\
$\quad$ injection beam & \\
Momentum spread of the injection beam & $\pm 0.1 \%$ \\
Extraction energy & $3 \mathrm{GeV}$ \\
Repetition rate & $25 \mathrm{~Hz}$ \\
Acceleration time & $20 \mathrm{~ms}$ \\
Ramping pattern & Sinusoidal \\
Transition energy & $9.2 \mathrm{GeV}$ \\
Harmonic number & 2 \\
Number of bunches & 2 \\
Number of particles per pulse & $8.33 \times 10^{13}$ \\
Output beam power & $1 \mathrm{MW}$ \\
Momentum acceptance & $\pm 1 \%$ \\
Ring acceptance & $486 \pi \mathrm{mm} \mathrm{mrad}$ \\
Collimator aperture & $324 \pi \mathrm{mm} \mathrm{mrad}$ \\
Collimator capability & $4 \mathrm{~kW}$ \\
Betatron tunes & $(6.45,6.42)$ or its \\
& $n e i g h b o r s$ \\
\hline \hline
\end{tabular}


$\left(\gamma_{t}=9.2 \mathrm{GeV}\right)$, that is sufficiently far from the extraction energy. This high- $\gamma_{t}$ lattice obtained by the missing-bend technique is a unique feature of the RCS optics design. Three families of sextupole magnets for chromatic correction are installed in the high-dispersion areas. On the other hand, the straight insertions have zero dispersion. Injection and collimation systems are installed in the first straight section; the injection system uses the first 1.5 cells, while the transverse primary collimator (scatterer) and the secondary collimators (absorbers) use the remainder of the cells. Extraction and rf systems are allocated in the second and the third straight sections.

The ring acceptance of RCS is $486 \pi \mathrm{mm} \mathrm{mrad}$ for a possible momentum spread of $\pm 1 \%$, for which the primary collimator aperture is set to $324 \pi \mathrm{mm}$ mrad. Such a large ring acceptance is essential for restricting particle losses within a permissible level. Also, such a large ratio (1.5) of the ring acceptance to the collimator aperture is required for localizing the residual particle losses in the collimator section and for minimizing the irradiation to the remainder of the ring [5]. The capacity of the collimator system is $4 \mathrm{~kW}$, which corresponds to $3 \%$ beam loss at injection assuming the 1-MW design beam operation.

As mentioned in the last section, the injector linac upgrades have been completed recently; the output energy of the linac beam was upgraded from $181 \mathrm{MeV}$ to the design value of $400 \mathrm{MeV}$ in 2013 by adding an annular-ring coupled structure (ACS) linac [6], and then the peak current of the linac beam was increased from $30 \mathrm{~mA}$ to the design value of $50 \mathrm{~mA}$ in 2014 by replacing the front-end system (ion source [7] and rf quadrupole [8]). Via these series of injector linac upgrades in 2013 and 2014, all hardware parameters to realize the RCS design output beam power of $1 \mathrm{MW}$ were in place.

\section{OUTLINE OF NUMERICAL SIMULATION}

In RCS, numerical simulation is performed mainly by using a particle tracking code called "Simpsons" developed by Shinji Machida [9]. This code enables us to simulate the three-dimensional motion of beam particles including the space-charge effect and the realistic injection process. We have been using this code since the design stage of RCS, and continuing its improvement to realize more realistic numerical simulation. The numerical simulation now plays an essential role in understanding beam loss mechanism and finding its solution, in combination with actual beam experiments.

In this code, all lattice elements are represented as thin lens elements. The conversion from thick lens to thin lens description is performed with another code "Teapot" [10]. At this stage, time-independent lattice imperfections such as field and alignment errors are reflected in the thin lens representation.

Our numerical simulation includes all lattice imperfections, that have been identified so far $[3,11]$; (a) Time-independent lattice imperfections (i) Multipole field components of main ring magnets, (ii) Magnetic field errors among same-family magnets, (iii) Misalignments, (b) Time-dependent lattice imperfections (i) Static leakage fields from extraction beam-line magnets, (ii) Edge focuses of injection bump magnets, (iii) $1-\mathrm{kHz}$ field ripples of main dipole magnets, (iv) $100-\mathrm{kHz}$ dipole field ripple induced by injection bump field, (v) Field tracking errors among main dipole and quadrupole magnet families. They were evaluated through magnetic field measurements and/or beam-based measurements. One of the characteristic features of this code is to take "time" as an independent variable, not longitudinal position, which is used more widely. Therefore, time-dependent lattice imperfections (b), as well as time-independent ones (a), can be implemented easily in the simulation. As is shown later, some of the lattice imperfections, coupled with the space-charge and the image-charge effects, cause beam loss.

This code can also simulate foil scattering during chargeexchange injection with scattering angle distributions calculated by "Geant" [12] assuming both Coulomb and hadronic interactions beforehand. Foil scattering beam loss is inevitable as long as a charge-exchange foil is used.

This code computes the space-charge potential based on a particle-in-cell algorithm with cylindrical meshes in the $(r, z, \varphi)$ coordinates. In the cylindrical coordinates, the Poisson equation is expressed as,

$$
\frac{1}{r} \frac{\partial}{\partial r}\left(r \frac{\partial}{\partial r} \phi\right)+\frac{1}{r^{2}} \frac{\partial^{2}}{\partial \varphi^{2}} \phi=-\frac{\rho}{\epsilon_{0}},
$$

considering the following ordering,

$$
\frac{\partial^{2}}{\partial z^{2}} \phi \sim \epsilon_{0} \mu_{0} \frac{\partial^{2}}{\partial t^{2}} \phi \ll \frac{1}{r} \frac{\partial}{\partial r}\left(r \frac{\partial}{\partial r} \phi\right)+\frac{1}{r^{2}} \frac{\partial^{2}}{\partial \varphi^{2}} \phi .
$$

Once a charge distribution at the cylindrical grid points is obtained, it is Fourier-decomposed in the azimuthal direction,

$$
\begin{gathered}
\phi(r, z, \varphi)=\sum_{m=-\infty}^{\infty} \phi_{m}(r, z) e^{i m \varphi}, \\
-\frac{\rho}{\epsilon_{0}} \equiv n(r, z, \varphi)=\sum_{m=-\infty}^{\infty} n_{m}(r, z) e^{i m \varphi} .
\end{gathered}
$$

The space-charge potential is solved separately in each mode as,

$$
\frac{1}{r} \frac{\partial}{\partial r}\left(r \frac{\partial}{\partial r} \phi_{m}(r, z)\right)-\frac{m^{2}}{r^{2}} \phi_{m}(r, z)=n_{m}(r, z),
$$

with the boundary condition of a circular cross-sectional beam pipe. The number of azimuthal modes is typically 
taken up to $m=8$. The space-charge potential is recalculated at a given time step out of the macroparticles whose distribution evolves in a self-consistent manner with the progression of time. Here, the space-charge kick is applied to each macroparticle as an integrated value considering the contribution of the magnetic field with a factor of $1 / \gamma^{2}$ at the end of each time step. The time step applied in the present simulation was 2 ns during injection and $10 \mathrm{~ns}$ during other processes, which correspond to 850 and 170 kicks per turn.

The number of macroparticles was typically set to $5 \times 10^{5}$ using a transverse grid of $64(r) \times 64(\varphi)$ for a conducting boundary of $r=0.145 \mathrm{~m}$ and a longitudinal grid of $50(z)$. Careful analyses for the convergence of macroparticle motions confirmed that the use of these numerical parameters is tolerable for obtaining meaningful simulation results [13].

\section{HIGH-INTENSITY BEAM TESTS OF UP TO $539 \mathrm{~kW}$ CONDUCTED BEFORE INJECTION ENERGY UPGRADE}

In November 2012, before the injection energy upgrade, we performed a $539-\mathrm{kW}$ high-intensity beam test with an injection energy of $181 \mathrm{MeV}$ using a $0.5 \mathrm{~ms}$-long injection pulse with a peak current of $24.3 \mathrm{~mA}$ and a chopper beamon duty factor of $60 \%$, where the number of particles per pulse totaled $4.5 \times 10^{13}$. In this beam test, the operational bare tune was set to $(6.45,6.42)$, which enables incoherent tune shifts to avoid serious systematic betatron resonances.

\section{A. Beam loss mitigation by injection painting}

In high-power proton machines such as RCS, the direct space-charge effect in the low-energy region is one of the most crucial sources of beam loss, imposing a strong limit on the achievable beam intensity. To alleviate this, RCS adopted a multiturn charge-exchange injection painting scheme. This approach permits us to control the charge density distribution of the circulating beam in both the transverse and the longitudinal phase spaces. In this beam test, we investigated the effectiveness of injection painting for space-charge mitigation.

\section{Transverse injection painting}

Transverse injection painting [13] makes use of a controlled phase-space offset between the centroid of the injection beam and the ring closed orbit to form a different particle distribution of the circulating beam from the multiturn injected beam.

Figure 3 shows a schematic view of the RCS injection section. For beam injection, two types of orbit-bump systems are prepared. One is the shift-bump system (four horizontal pulse dipole magnets; SB1 -4) to produce a flat-top field during injection, making a horizontal orbit bump offset at the injection point where the chargeexchange foil is installed. Another is the paint-bump system (four horizontal and two vertical pulse dipole magnets; PBH1 - 4 and PBV1-2) to make a time dependent bump orbit at the injection point for beam painting.

Figure 4 shows a schematic diagram of injection painting on the horizontal and the vertical phase spaces. In the horizontal painting process, PBHs, which are installed in the ring, produce a horizontal closed orbit variation at the injection point, by which the injected beam is distributed from the middle to the outside in the circulating beam ellipse along its major axis. On the other hand, in the vertical painting process, $\mathrm{PBVs}$, which are installed in the

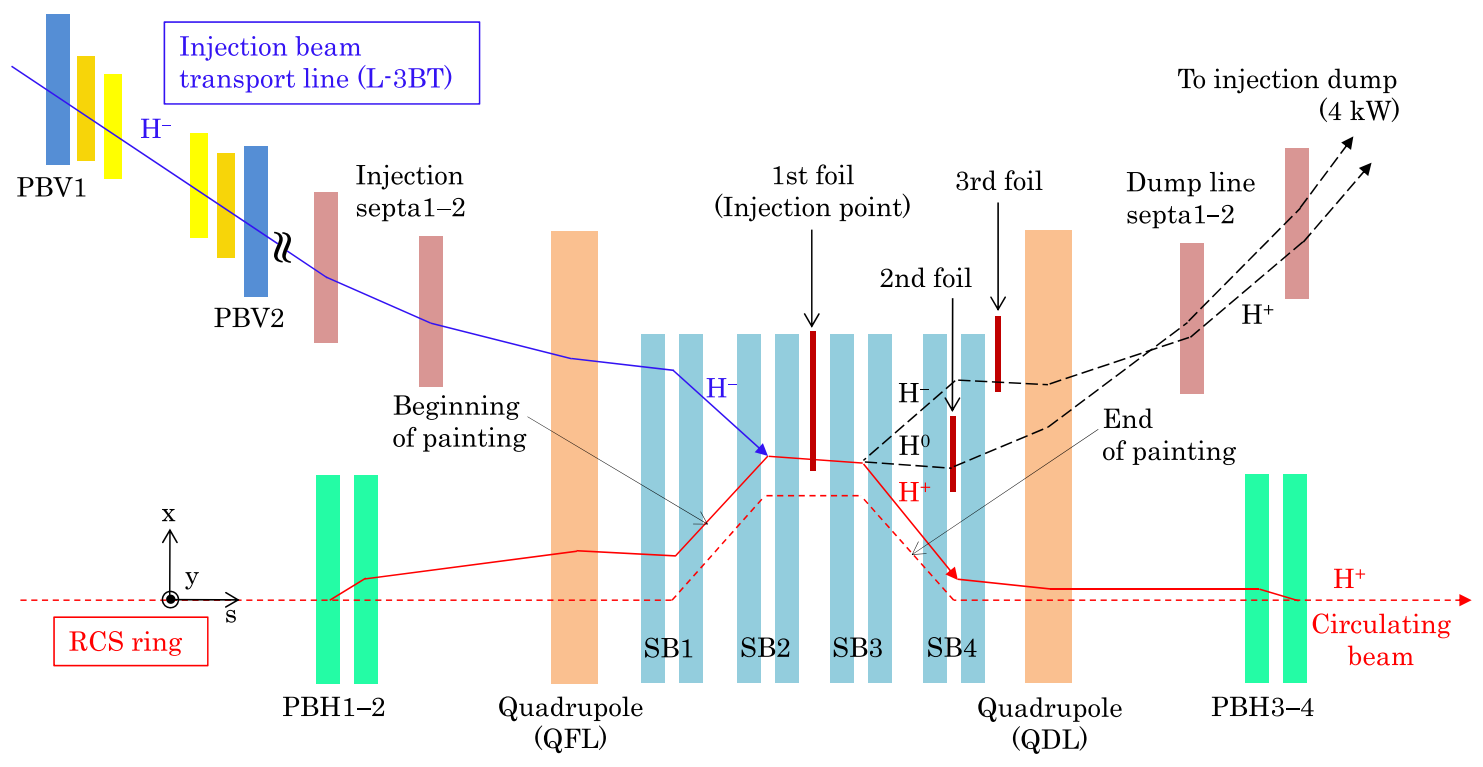

FIG. 3. Schematic view of the injection section. 


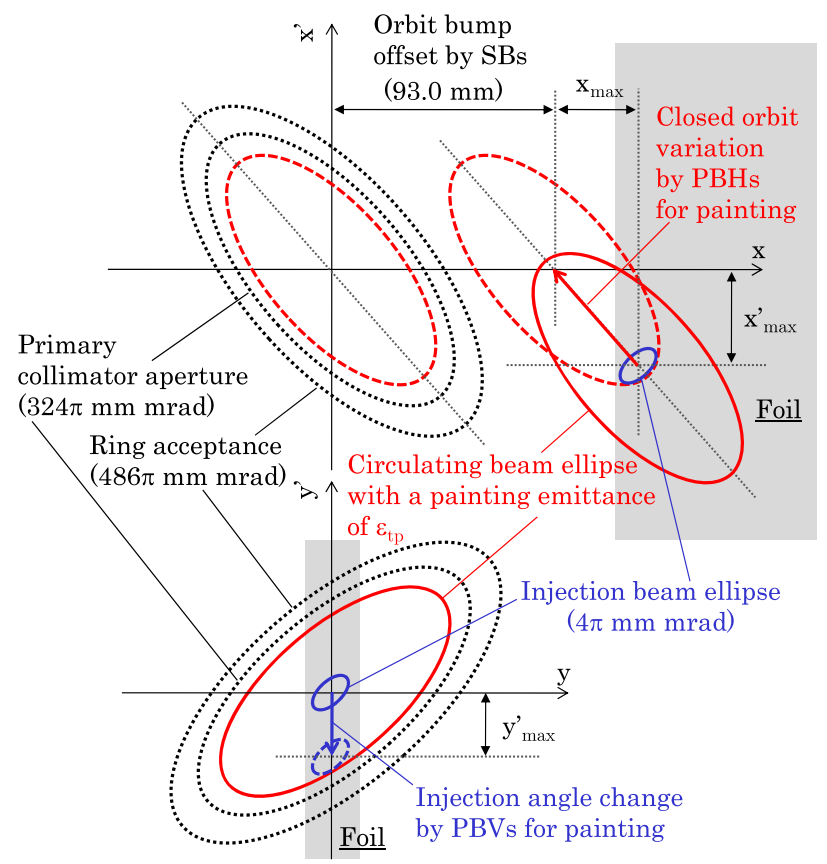

FIG. 4. Schematic diagram of transverse injection painting, where the upper one corresponds to the horizontal plane, and the lower one to the vertical plane.

injection beam transport line nearly 180-degrees upstream of the injection point in betatron phase advance, produce a vertical injection angle change at the injection point, by which the injected beam is similarly filled from the middle to the outside in the circulating beam ellipse along its angular axis.

For this painting process, the phase-space offset of the injection beam relative to the ring closed orbit is varied as per the square-root-type functions;

$$
\begin{gathered}
x_{\text {paint }}=x_{\max } \sqrt{t / T}, \quad x_{\text {paint }}^{\prime}=-x_{\max }^{\prime} \sqrt{t / T}, \\
y_{\text {paint }}=0, \quad y_{\text {paint }}^{\prime}=-y_{\max }^{\prime} \sqrt{t / T} .
\end{gathered}
$$

Here, $\left(x_{\max }, x_{\max }^{\prime}\right)$ and $\left(0, y_{\max }^{\prime}\right)$ are the maximum phasespace offsets on the horizontal and the vertical planes, for which the injection beam ellipse is inscribed at the edge of the circulating beam ellipse with the required painting emittance $\epsilon_{t p}$. The other parameters are: $T$ represents an injection duration of $0.5 \mathrm{~ms}$, and $t$ indicates a time step from 0 through the end of injection $T$. This type of transverse painting is called correlated painting [14,15]. In this experiment, the painting emittance was set to $\epsilon_{t p}=100 \pi \mathrm{mmmrad}$, which corresponds to $x_{\max }=$ $27.1 \mathrm{~mm}, x_{\max }^{\prime}=3.8 \mathrm{mrad}$ and $y_{\max }^{\prime}=2.3 \mathrm{mrad}$.

As shown in Fig. 5, the charge density peak in the transverse direction is well reduced by this transverse injection painting.
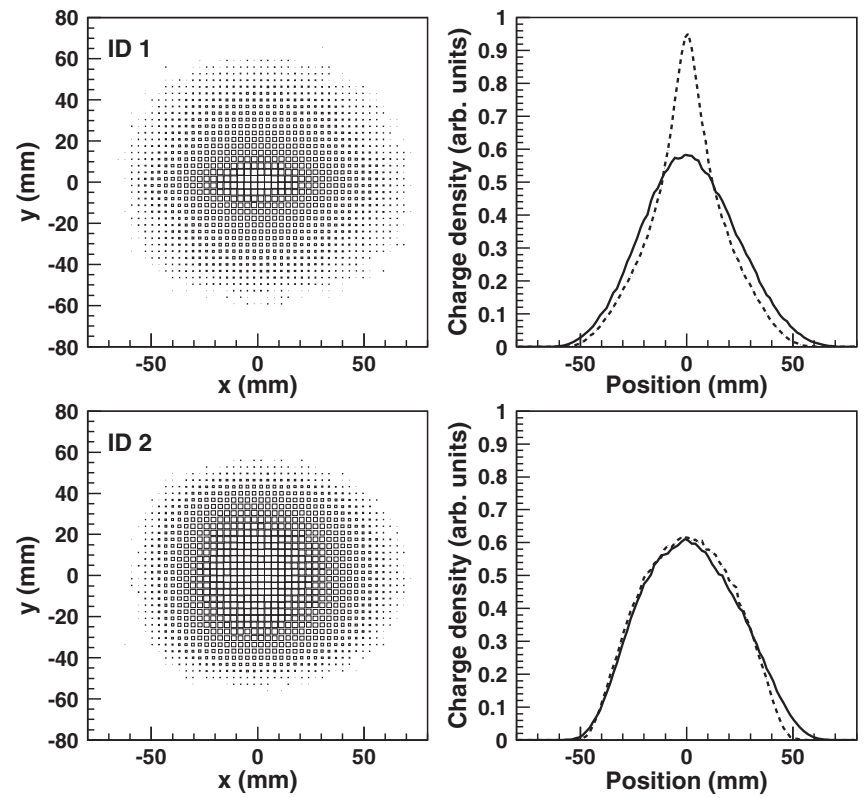

FIG. 5. Particle distributions on the two-dimensional transverse plane $(x, y)$ and their projections onto the $x$ (solid curves) and the $y$ (dashed curves) axes, calculated at the end of injection on the foil for the beam intensity of $539 \mathrm{~kW}$ with the painting parameter IDs 1 and 2 in Table II given later.

\section{Longitudinal injection painting}

Longitudinal injection painting [16,17] makes use of a controlled momentum offset to the rf bucket in combination with superposing a second harmonic rf to obtain a uniform longitudinal particle distribution after the multiturn injection.

Figure 6 shows a schematic diagram of the momentum offset injection. As shown in the figure, a flat beam bunch is formed through emittance dilution due to a large synchrotron motion excited by the momentum offset. In this

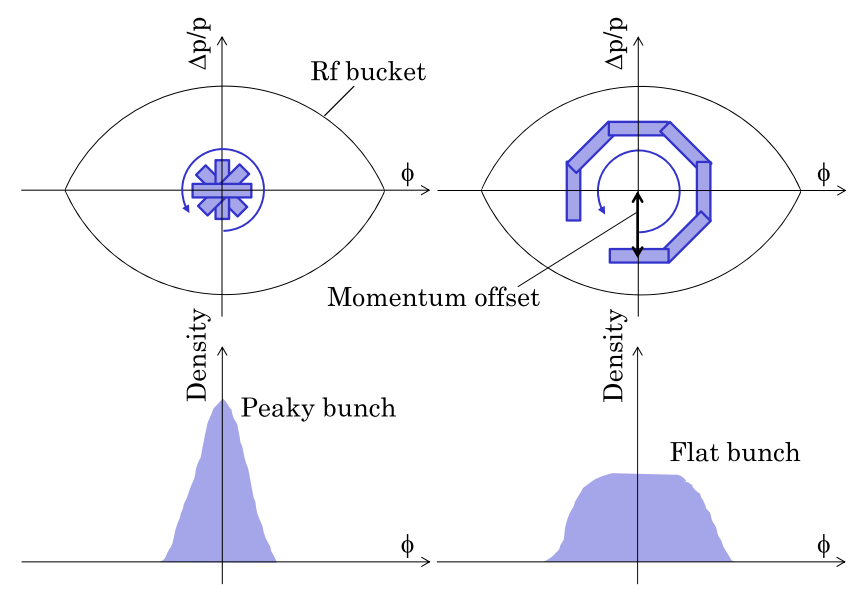

FIG. 6. Schematic diagram of the longitudinal motion for the multiturn injection process without (left) and with (right) a momentum offset, where the boxes represent the injection bunch train from the linac. 


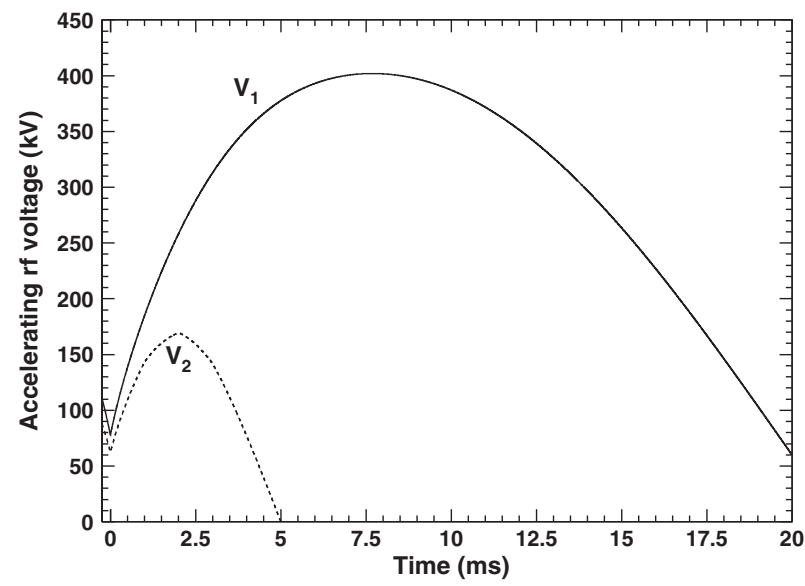

FIG. 7. Fundamental $\left(V_{1}\right)$ and second $\left(V_{2}\right)$ harmonic rf voltage patterns over the acceleration period.

method, the superposition of a second harmonic rf plays an important role in shaping a flatter and wider rf bucket potential, helping to create a flatter bunch distribution. As an additional control in longitudinal painting, a phase sweep of the second harmonic rf was also employed. This phase sweep enables further bunch distribution control through a dynamical change of the rf bucket potential shape including a position change of the stable fixed points during injection.

The rf voltage $V_{\text {rf }}$ applied in the experiment is expressed as,

$$
V_{\mathrm{rf}}=V_{1} \sin \phi-V_{2} \sin \left\{2\left(\phi-\phi_{s}\right)+\phi_{2}\right\},
$$

where $V_{1}$ is the amplitude of the fundamental rf, $V_{2}$ is the amplitude of the second harmonic rf, $\phi_{s}$ is the synchronous phase, $\phi$ is the phase of the fundamental rf voltage, and $\phi_{2}$ is the phase offset of the second harmonic rf. In the experiment, the second harmonic rf with an amplitude of $80 \%\left(V_{2} / V_{1}\right)$ of the fundamental rf was employed during the first $1 \mathrm{~ms}$, as shown in Fig. 7. Also, its phase was swept linearly from -100 to 0 degrees $\left(\phi_{2}\right)$ over an injection duration of $0.5 \mathrm{~ms}$. In this case, the shape of the rf bucket potential gradually changes during injection, as shown in Fig. 8. For such a dynamically controlled rf bucket potential, momentum offset injections of $0,-0.1$, and $-0.2 \%(\Delta p / p)$ were tested.

As shown in Fig. 9, the charge density peak in the longitudinal direction considerably decreases following the progression of longitudinal painting.

\section{Injection painting parameter dependence of beam loss}

Figure 10 shows the time dependence of the circulating beam intensity from injection through extraction, measured with systematic combinations of transverse and longitudinal painting listed in Table II. For the parameter

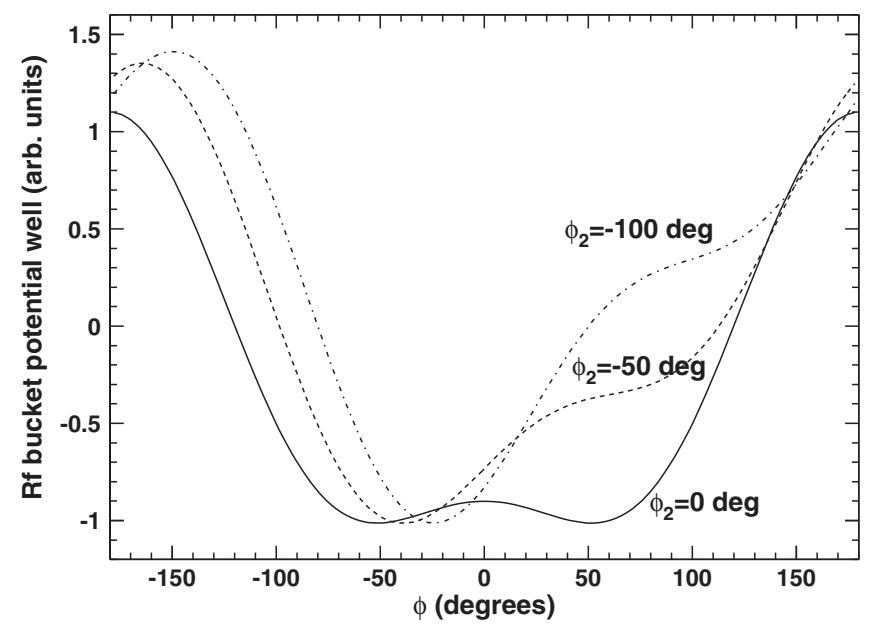

FIG. 8. Time dependence of the rf bucket potential well during injection.

ID 1 with no painting, a considerable $31.1 \%$ beam loss was observed in the low-energy region, where the spacecharge effect is most serious. But, this beam loss was drastically mitigated from ID 1 to ID 5 by longitudinal painting, and from ID 5 to ID 8 by adding transverse painting; the beam loss was finally reduced to $1.6 \%$ by ID 8 .

These observations were well reproduced by the corresponding numerical simulations, as shown by the dashed red curves in the figure. Figure 11 shows the tune footprints calculated at the end of injection without (ID 1) and with (ID 8) injection painting. In the left plot obtained with ID 1, one can see that a core part of the beam particles crosses various low-order systematic resonances due to large spacecharge detuning. Such particles on the resonances suffer from emittance dilutions. This is the main cause of the large beam loss observed for ID 1 . As shown in the right plot, the space-charge tune shift is well mitigated through the charge distribution control by ID 8. This acts to mitigate the effect of the resonances, leading to the significant beam loss reduction from ID 1 to ID 8 .

These beam experiments and numerical simulations not only confirmed the excellent ability of injection painting for space-charge mitigation, but also revealed an important view on the space-charge limit. As is well known, the strength of the space-charge effect is simply indicated by the Laslett tune shift [18];

$$
\Delta \nu=-\frac{n_{t} r_{p}}{2 \pi \beta^{2} \gamma^{3} \epsilon} \frac{1}{B_{f}},
$$

where $n_{t}$ is the total number of protons per pulse, $r_{p}$ the classical radius of proton, $\beta$ and $\gamma$ the Lorenz factors, $\epsilon$ the transverse emittance, and $B_{f}$ the bunching factor. The Laslett tune shift for ID 8 in this beam test was calculated to be -0.484 at the injection energy of $181 \mathrm{MeV}$. This corresponds to 1.6 times the value of the Laslett tune shift at the injection energy of $400 \mathrm{MeV}$ for the 1-MW design 

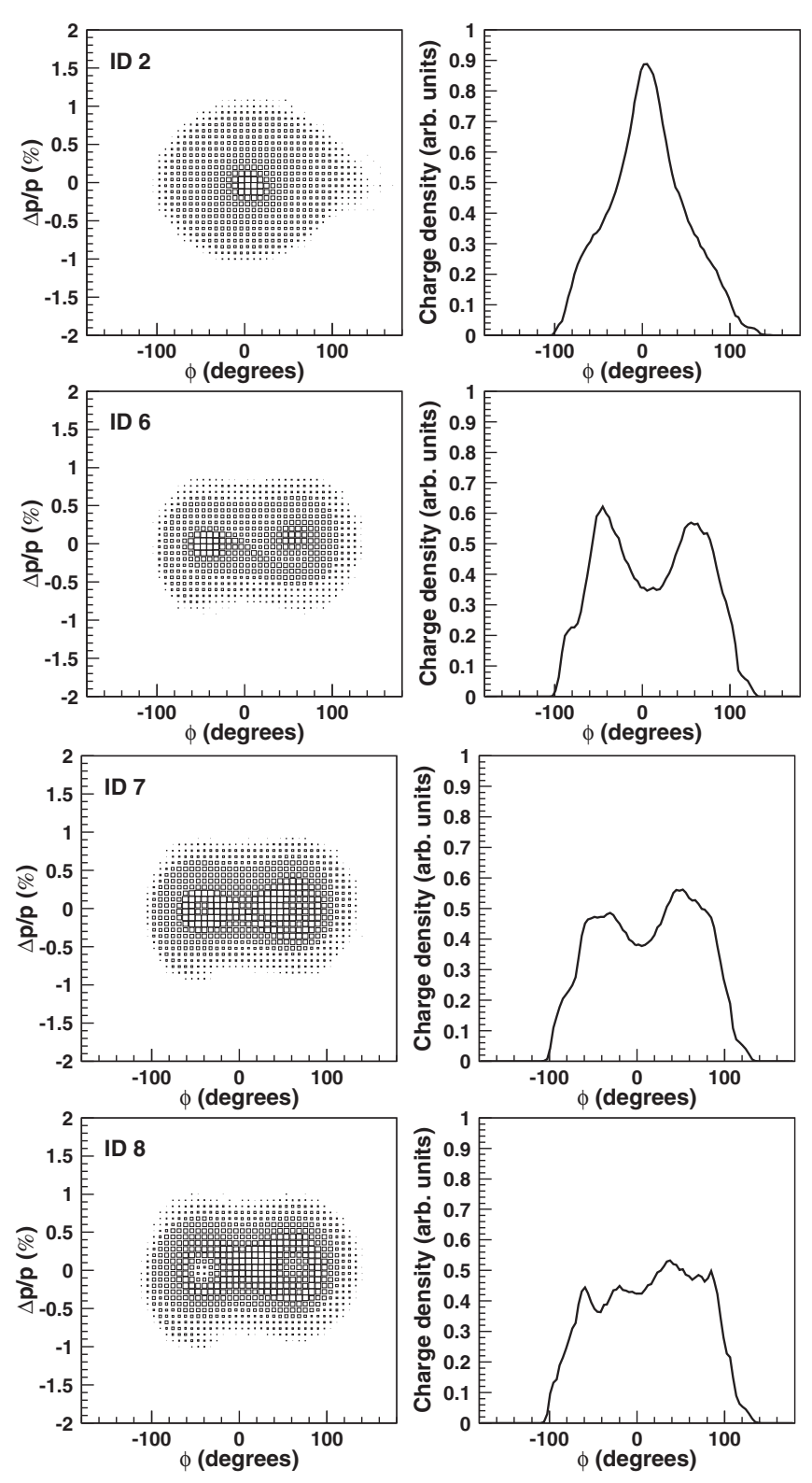

FIG. 9. Particle distributions on the longitudinal phase space $(\phi, \Delta p / p)$ and their projections onto the $\phi$ axis, calculated at the end of injection for the beam intensity of $539 \mathrm{~kW}$ with the painting parameter IDs 2, 6, 7 and 8 in Table. II given later.

beam operation. In other words, the space-charge strength is equivalent to that for the 1.6-MW beam operation. Although the beam intensity in this experiment is around half of the design value, the space-charge strength is considerably higher as per the $1 / \beta^{2} \gamma^{3}$ scaling law owing to the lower injection energy of $181 \mathrm{MeV}$. The fact that sufficient beam loss reduction was achieved under such a severe condition implies that RCS is well suited for realizing the design output beam power of $1 \mathrm{MW}$ after the injection energy upgrade, and also it has potential for further beam power ramp-up to $1.6 \mathrm{MW}$ or more from the viewpoint of the space-charge limit.

\section{B. Beam loss caused by $100-\mathrm{kHz}$ dipole field ripple and its mitigation}

As mentioned in the last subsection, the beam loss for the beam intensity of $539 \mathrm{~kW}$ was drastically reduced from $31.1 \%$ to $1.6 \%$ by introducing injection painting of ID 8. In order to realize the cause of the residual beam loss and its further mitigation, we then investigated the detailed time structure of beam loss and its beam intensity dependence.

\section{Time structure of residual beam loss and its beam intensity dependence}

The left plot in Fig. 12 shows the scintillation-type beam loss monitor (BLM) signals for the first $5 \mathrm{~ms}$ measured at the collimator section with the injection painting parameter of ID 8 for various beam intensities from 104 to $539 \mathrm{~kW}$, where the beam intensity was varied by changing the injection pulse length from 0.1 to $0.5 \mathrm{~ms}$. In this figure, one can look at the time structure of beam loss and its beam intensity dependence in more detail, thanks to the excellent time resolution of the scintillation-type BLM.

As shown in the figure, the time structure of beam loss shows a characteristic beam intensity dependence; the beam loss has two bump structures for the higher beam intensity, of which the second bump structure gradually vanishes in going to the lower beam intensity.

The source of the first bump structure of beam loss is obvious, which is mainly foil scattering during chargeexchange injection. This part of beam loss is inevitable as long as a charge-exchange foil is used, and thus it appears during injection for all the cases in proportion to the product of the beam intensity and the foil hitting rate. On the other hand, the second bump structure of beam loss is generated through a more complex mechanism arising from a dipole field ripple during injection, as is discussed later.

As shown in the right plot in Fig. 12, the empirical beam loss was well reproduced by the numerical simulation by taking into account the dipole field ripple. This numerical simulation provided consistent results also as to the other experimental data such as the transverse beam profile (Fig. 13) and the bunching factor (Fig. 14). With this well-established simulation result, we will discuss in detail the mechanism of the second bump structure of beam loss caused by the dipole field ripple.

\section{Source of dipole field ripple}

As shown in Fig. 3, beam injection is performed with four sets of pulse-type injection bump magnets SB1 - 4 connected in series. In SBs, rf-shielded ceramic chambers are installed. Figure 15 shows a schematic drawing of the chamber, where the brown lines are the rf shield stripes made of $\mathrm{Cu}$, and these $\mathrm{Cu}$ stripes are terminated at either end by capacitors. The dipole field ripple 


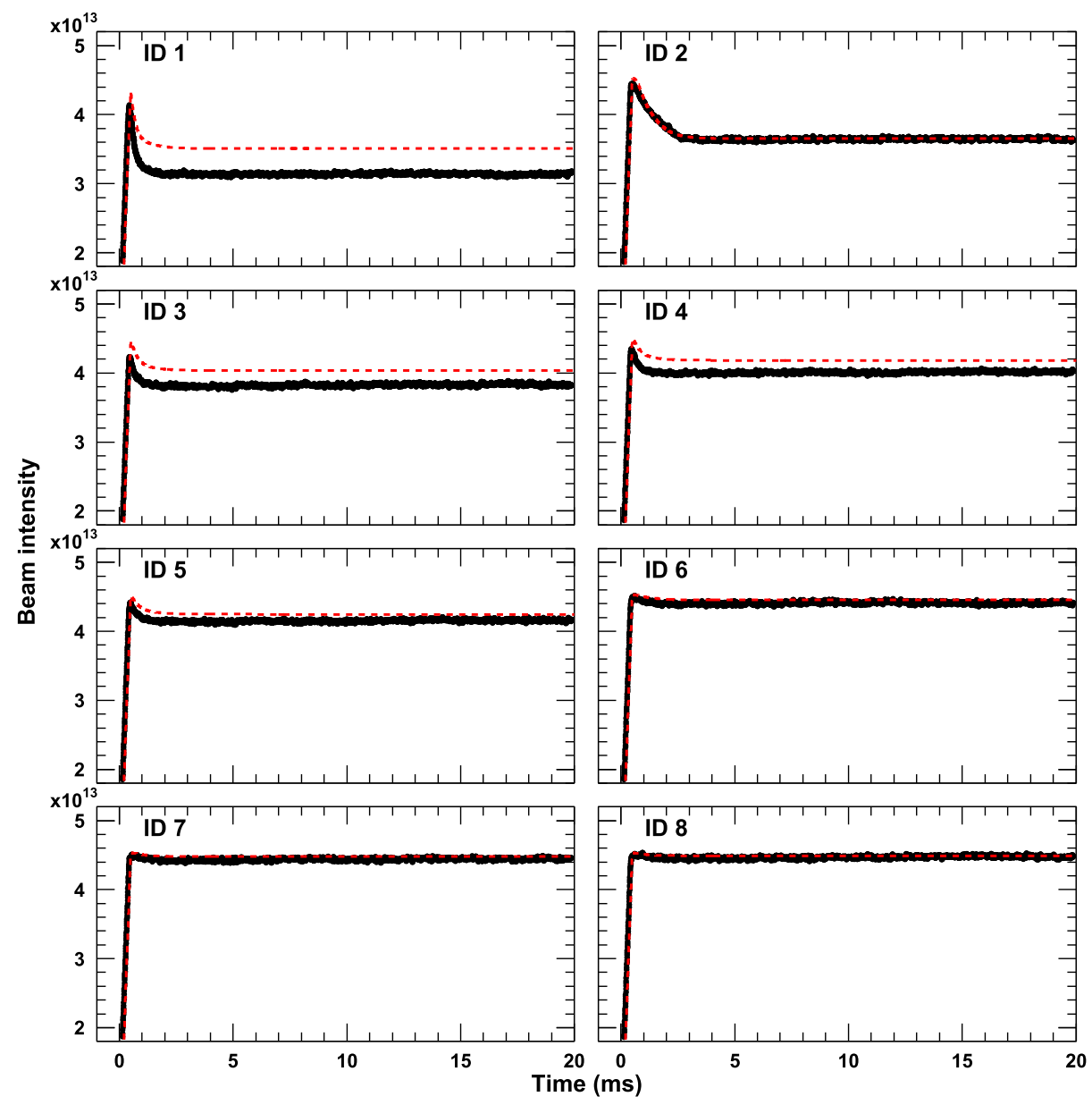

FIG. 10. Time dependence of the circulating beam intensity from injection through extraction, measured with the systematic combinations of transverse and longitudinal injection painting of IDs 1-8 in Table II, where the dashed red curves show the corresponding numerical simulation results.

originates from resonant currents in the rf shield loops induced by the continuous ripple noise of the SBs field [19].

The power supply of SBs is based on a conventional insulated-gate bipolar transistor (IGBT) chopping system [20]. Therefore this power supply produces continuous ripple noise due to continuous switching throughout field formation. This effect of the SBs field itself is canceled through integration over the four SB magnets. Therefore it hardly affects the beam. The dipole field ripple coming from the resonant currents induced in the rf shield loops can also be canceled out similarly if the rf-shield loops have a symmetric configuration. However, the symmetric condition was lost at the time, because some of the capacitors were out of order owing to discharge. The residual dipole field ripple component due to such an asymmetric configuration affected the beam, that is, excited a coherent position oscillation.

The left plot in Fig. 16 shows the turn-by-turn coherent position oscillation for the first $1.3 \mathrm{~ms}$ measured with a beam position monitor (BPM) [21]. By analyzing the BPM data, the kick angle of the dipole field ripple was evaluated as a function of time, as shown in the right plot in Fig. 16. The dipole field ripple appears only for the first $1 \mathrm{~ms}$ when the injection bump is activated, and its frequency is around $100 \mathrm{kHz}$.

TABLE II. Injection painting parameters applied in the present beam experiment; (ID 1) no painting, (ID 2) transverse painting only, (IDs 3-5) longitudinal painting only, and (IDs 6-8) combinations of transverse and longitudinal painting.

\begin{tabular}{lccrc}
\hline \hline $\begin{array}{l}\text { Parameter } \\
\text { IDs }\end{array}$ & $\begin{array}{c}\epsilon_{t p} \\
(\pi \mathrm{mm} \text { mrad })\end{array}$ & $\begin{array}{c}V_{2} / V_{1} \\
(\%)\end{array}$ & $\begin{array}{c}\phi_{2} \\
(\text { degrees })\end{array}$ & $\begin{array}{c}\Delta p / p \\
(\%)\end{array}$ \\
\hline 1 & $\ldots$ & $\ldots$ & $\ldots$ & $\ldots$ \\
2 & 100 & $\ldots$ & $\ldots$ & $\ldots$ \\
3 & $\ldots$ & 80 & -100 to 0 & $\ldots$ \\
4 & $\ldots$ & 80 & -100 to 0 & -0.1 \\
5 & $\ldots$ & 80 & -100 to 0 & -0.2 \\
6 & 100 & 80 & -100 to 0 & $\ldots$ \\
7 & 100 & 80 & -100 to 0 & -0.1 \\
8 & 100 & 80 & -100 to 0 & -0.2 \\
\hline \hline
\end{tabular}



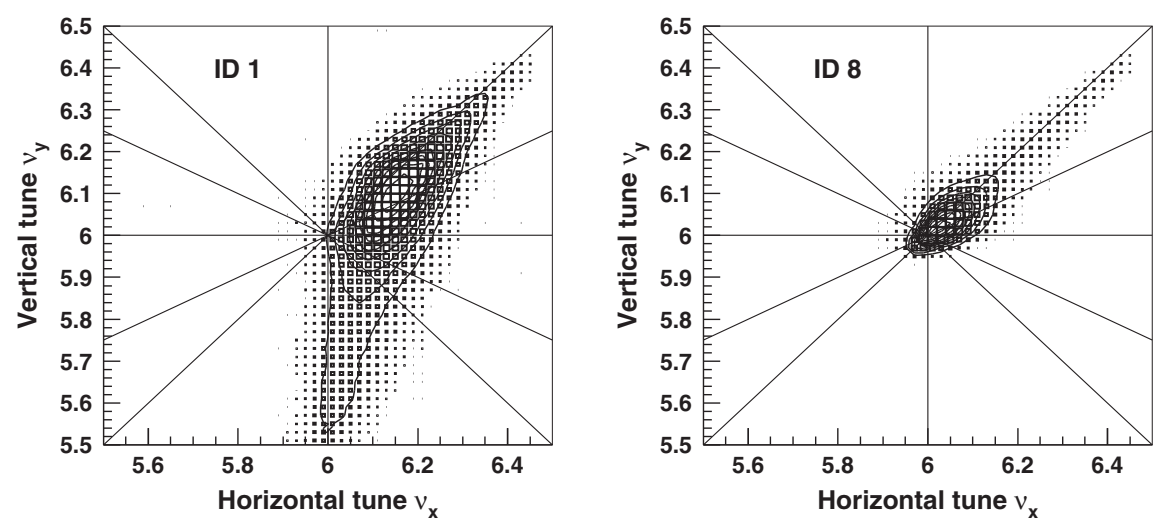

FIG. 11. Tune footprints at the end of injection calculated for the beam intensity of $539 \mathrm{~kW}$ without (ID 1) and with (ID 8) injection painting, where the solid lines show systematic resonances up to fourth order.

\section{Discussion for beam loss mechanism caused by dipole field ripple}

The numerical simulation well reproduced the empirical beam loss, as shown in Fig. 12, by taking into account the $100-\mathrm{kHz}$ dipole field ripple, and confirmed that the dipole field ripple is the main cause of the second bump structure in beam loss.

Figure 17 shows the transverse beam profiles at the end of injection calculated without and with the dipole field ripple. In this figure, one can see that the dipole field ripple forms a beam halo, and that the beam halo formation is more enhanced in going to the higher beam intensity. This beam halo causes the second bump structure of beam loss at the higher beam intensity.

The numerical simulation suggested that the imagecharge effect is key to understanding the mechanism of beam halo formation. Assuming a circular conducting boundary as shown in Fig. 18, which is a good
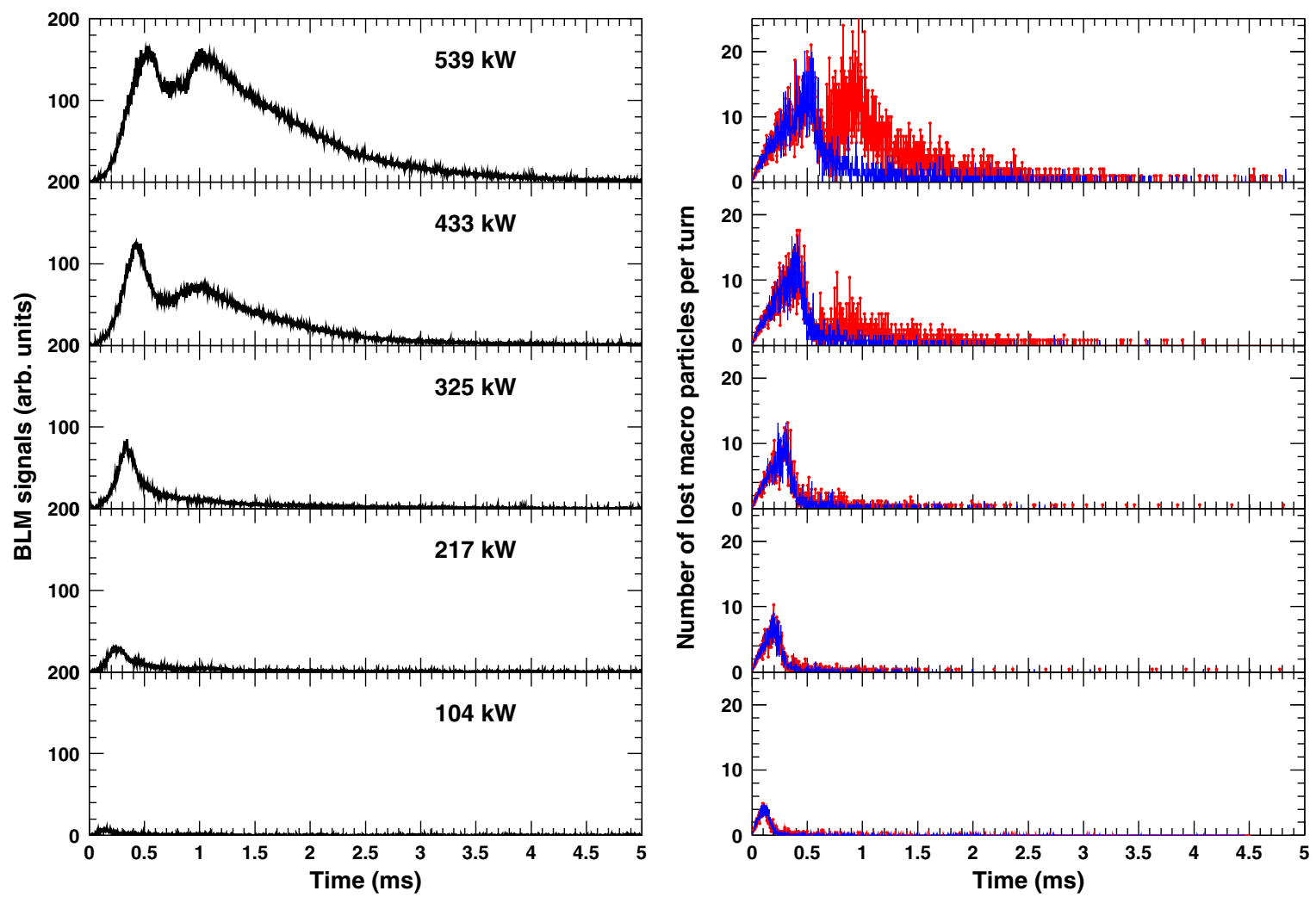

FIG. 12. (Left) Scintillation-type BLM signals for the first $5 \mathrm{~ms}$ measured at the collimator section with the injection painting parameter of ID 8 for various beam intensities from 104 to $539 \mathrm{~kW}$. (Right) Corresponding numerical simulation results obtained with (red) and without (blue) the dipole field ripple. 


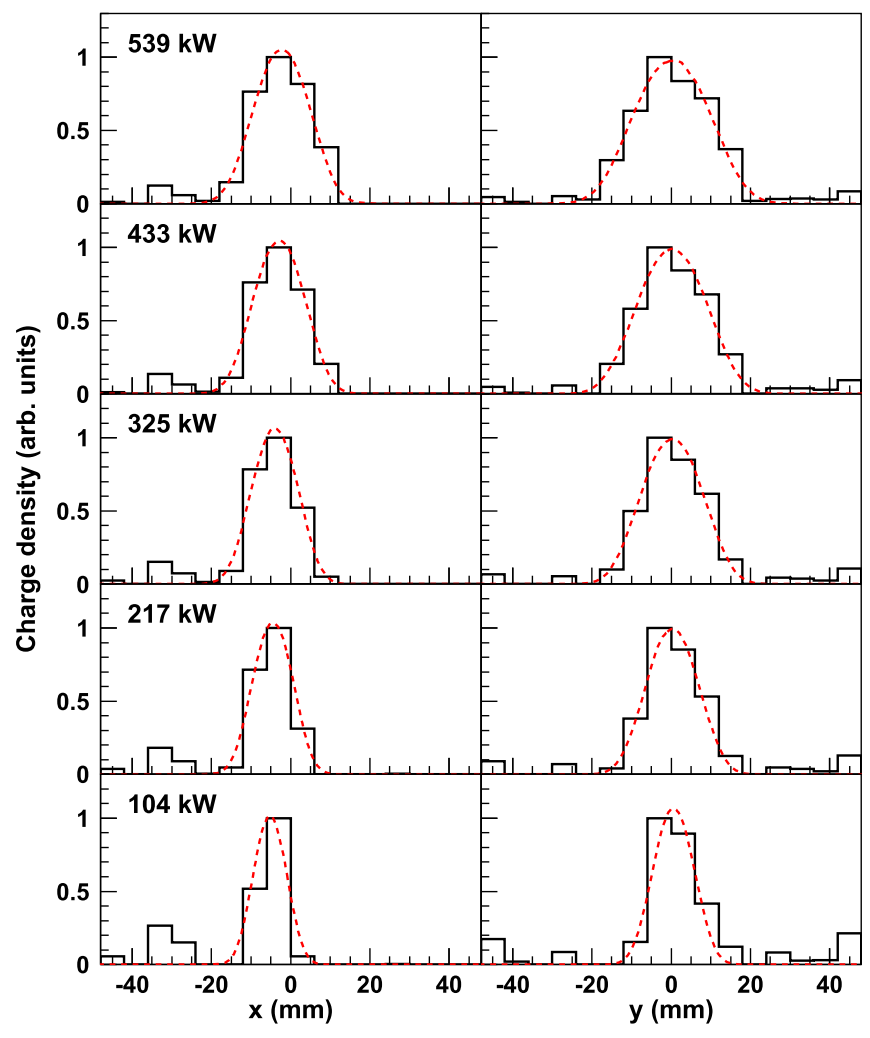

FIG. 13. Transverse beam profiles at $3 \mathrm{GeV}$ measured in the extraction beam transport line with the injection painting parameter of ID 8 for various beam intensities from 104 to $539 \mathrm{~kW}$, where the dashed red curves are the corresponding numerical simulation results.

approximation of RCS, the image-charge field is obtained as,

$$
\begin{array}{r}
E_{\chi}\left(\chi_{i}\right)=\frac{\lambda}{2 \pi \epsilon_{0}} \frac{1}{b-\chi_{i}}=\frac{\lambda}{2 \pi \epsilon_{0}}\left(\frac{\bar{\chi}}{\rho^{2}}+\frac{\bar{\chi}^{2} \chi_{i}}{\rho^{4}} \cdots\right), \\
E_{\chi}(\bar{\chi})=\frac{\lambda}{2 \pi \epsilon_{0}} \frac{1}{b-\bar{\chi}}=\frac{\lambda}{2 \pi \epsilon_{0}}\left(\frac{\bar{\chi}}{\rho^{2}}+\frac{\bar{\chi}^{3}}{\rho^{4}} \cdots\right) .
\end{array}
$$

Taking into account the above image-charge field, the equation of motion of the $i$ th particle is expressed as,

$$
\begin{aligned}
\chi_{i}^{\prime \prime}+k(s) \chi_{i}= & \sum_{j} F_{i j}^{s c}+f(s, t) \\
& +\frac{2 r_{p} \lambda}{e \beta^{2} \gamma}\left(\frac{\bar{\chi}}{\rho^{2}}+\frac{\bar{\chi}^{2} \chi_{i}}{\rho^{4}} \cdots\right),
\end{aligned}
$$

where $\chi$ represents the transverse position of $x$ or $y, s$ the longitudinal position, $k(s)$ the lattice focusing function, $F_{i j}^{s c}$ the space-charge force between the $i$ th and the $j$ th beam particles, $f(s, t)$ the dipole field ripple, and the third term on the right side of the equation is from the image charge. By averaging Eq. (12) for all particles and using

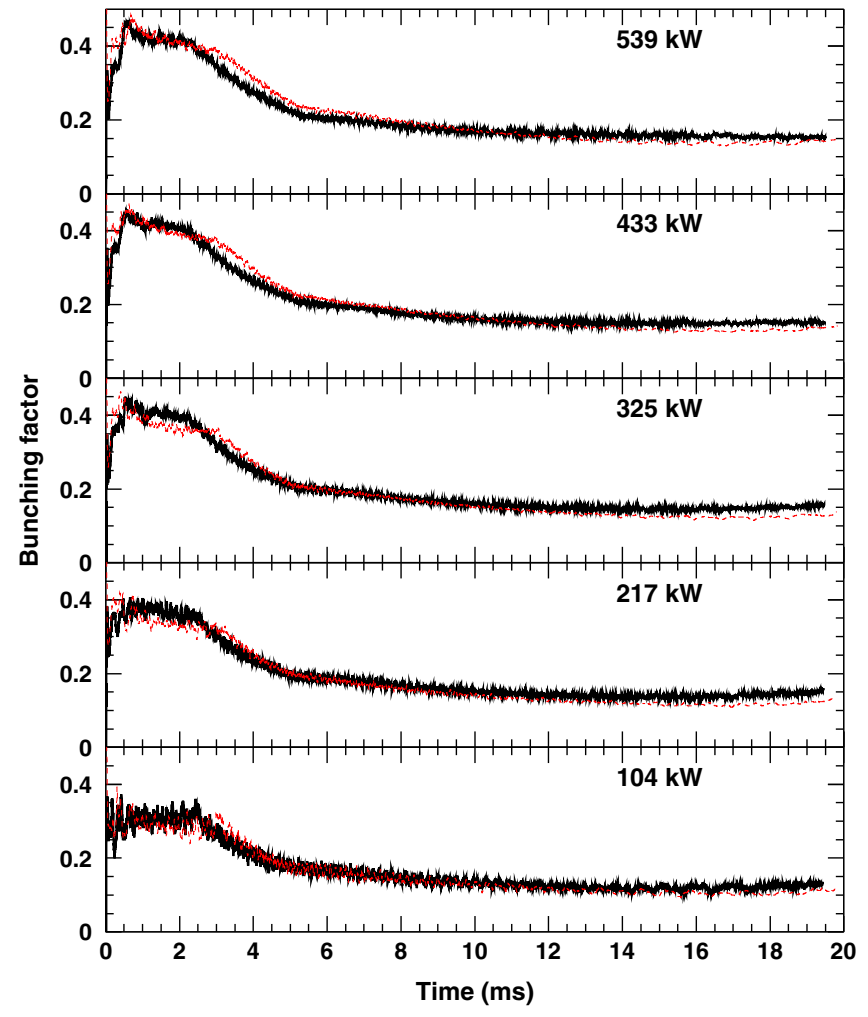

FIG. 14. Bunching factors measured with the injection painting parameter of ID 8 for various beam intensities from 104 to $539 \mathrm{~kW}$, where the dashed red curves are the corresponding numerical simulation results.

$F_{i j}^{s c}=-F_{j i}^{s c}$, the equation for coherent motion of beam $(\bar{\chi})$ is obtained as,

$$
\bar{\chi}^{\prime \prime}+k(s) \bar{\chi}=f(s, t)+\frac{2 r_{p} \lambda}{e \beta^{2} \gamma}\left(\frac{\bar{\chi}}{\rho^{2}}+\frac{\bar{\chi}^{3}}{\rho^{4}} \cdots\right),
$$

while the equation for incoherent motion of each particle $\left(\chi_{i}-\bar{\chi}\right)$ is found by subtracting Eq. (13) from Eq. (12) as,

$$
\begin{aligned}
& \left(\chi_{i}-\bar{\chi}\right)^{\prime \prime}+k(s)\left(\chi_{i}-\bar{\chi}\right) \\
& \quad=\sum_{j} F_{i j}^{s c}+\frac{2 r_{p} \lambda \bar{\chi}^{2}}{e \beta^{2} \gamma} \frac{\rho^{4}}{\rho^{4}}\left(\chi_{i}-\bar{\chi}\right) \cdots .
\end{aligned}
$$

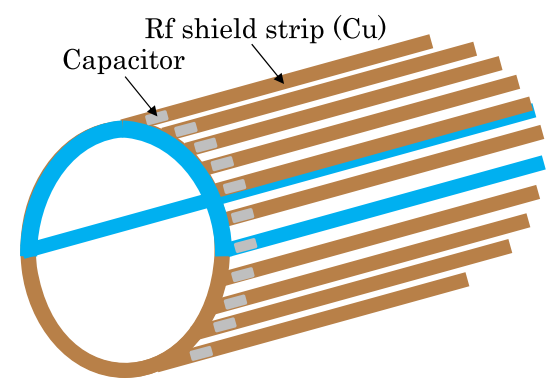

FIG. 15. Schematic drawing of the rf-shielded ceramic chamber. 

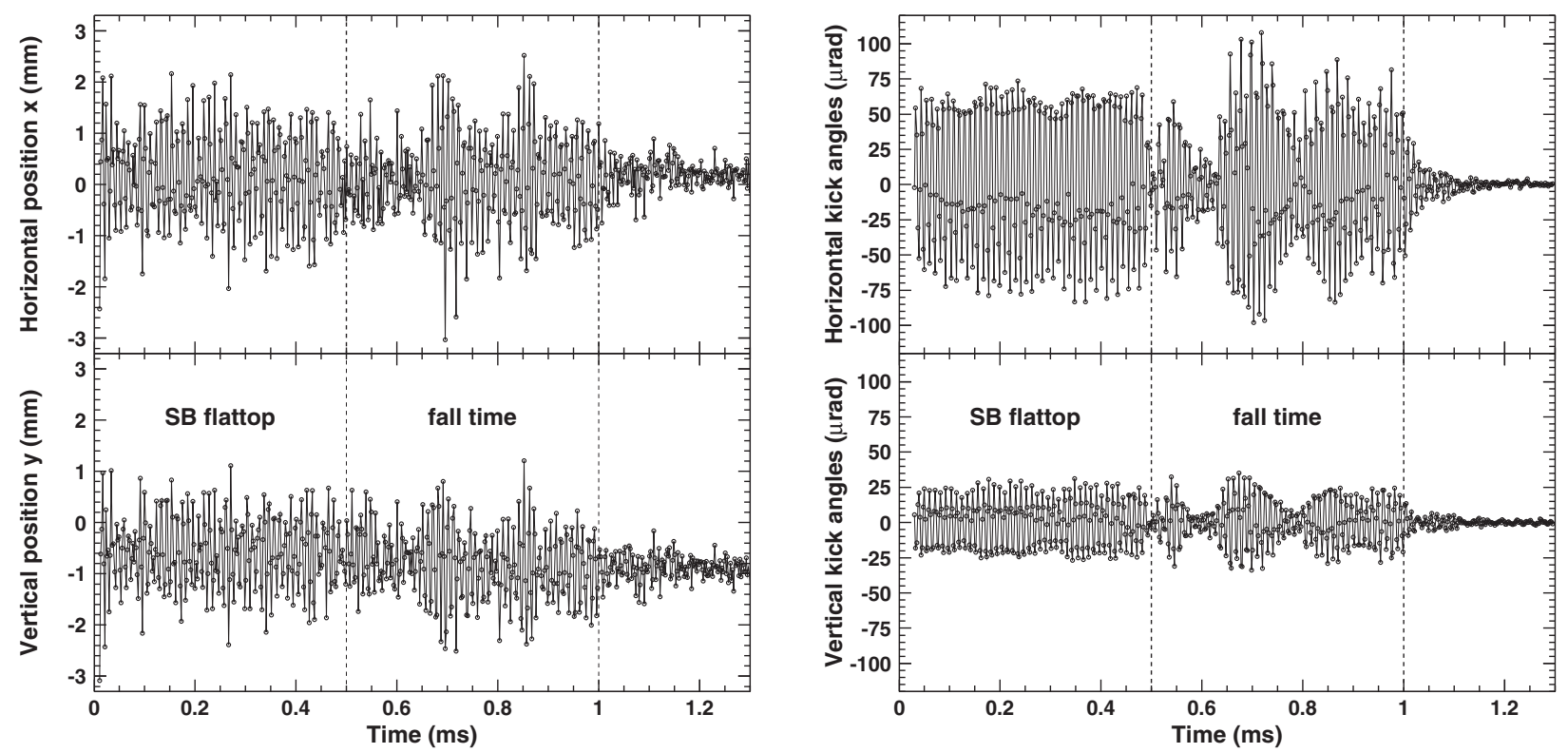

FIG. 16. (Left) Turn-by-turn coherent position oscillations for the first 1.3 ms measured with BPM. (Right) Kick angles of the dipole field ripple evaluated from the turn-by-turn BPM data.

The first term of the image-charge force contributing to the coherent motion of Eq. (13) is a simple quadrupole field proportional to $\bar{\chi}$. As is well known, this force causes a coherent tune shift depending on the beam intensity through $\lambda$. The first term of the image-charge force contributing to the incoherent motion of Eq. (14) is also a quadrupole field proportional to $\chi_{i}-\bar{\chi}$, but its strength changes through the coefficient of $\bar{\chi}^{2}$. Thus, if the coherent
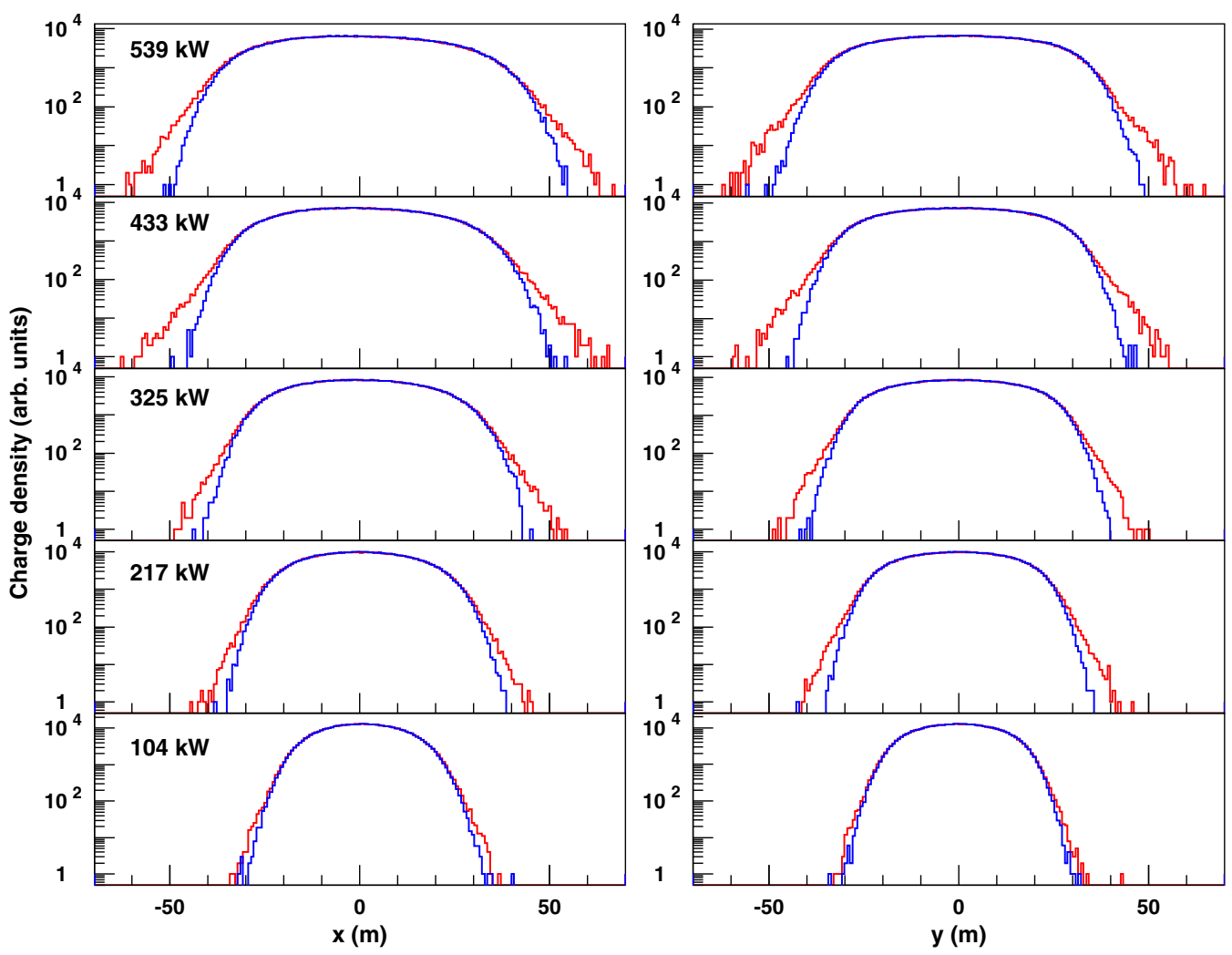

FIG. 17. Transverse beam profiles at the end of injection calculated with (red) and without (blue) the dipole field ripple for various beam intensities from 104 to $539 \mathrm{~kW}$. 


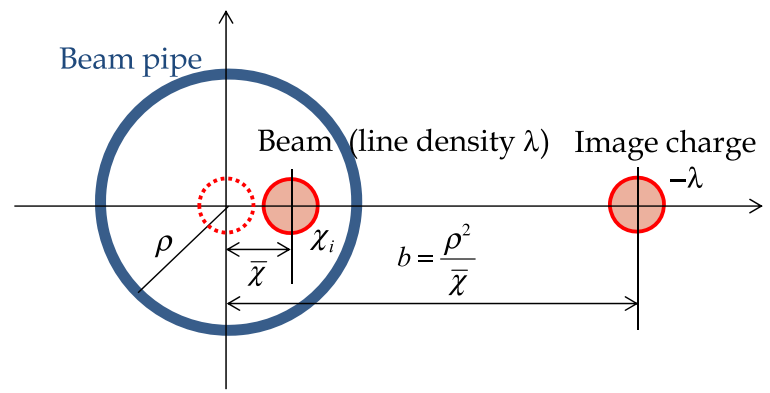

FIG. 18. Schematic diagram of the image-charge effect.

position oscillation is excited by some external force such as that considered herein, this quadrupole force acts to shake the beam envelope at twice the frequency of the coherent position oscillation. That is, it leads to a secondorder stopband at the corresponding incoherent betatron tune. The frequency of the coherent position oscillation observed in Fig. 16 is $100 \mathrm{kHz}$, which corresponds to 0.2 as the betatron tune. Accordingly, in the present case, the image-charge force in Eq. (14) is to drive the second-order resonance at $2 \delta \nu_{\chi}=0.4$, where $\delta \nu_{\chi}$ is the fractional part of the incoherent betatron tune.

In order to confirm the effect of the $2 \delta \nu_{\chi}=0.4$ resonance, we investigated the single-particle behavior for each macroparticle. Figure 19 shows the turn-by-turn incoherent motion of one macroparticle near $2 \delta \nu_{\chi}=0.4$. In this figure, one can find that the amplitude of the incoherent position oscillation is sharply enhanced when the incoherent betatron tune comes close to $2 \delta \nu_{\chi}=0.4$. As mentioned above, the bare betatron tune was set to $(6.45,6.42)$ in this beam test, which is far from the resonance, but some beam particles reach the resonance due to the space-charge tune depression. Such resonating beam particles suffer from emittance dilution as seen in Fig. 19, resulting in the beam halo observed in Fig. 17. The effect of the image charge depends on the beam intensity through $\lambda$. This is the main reason why the beam
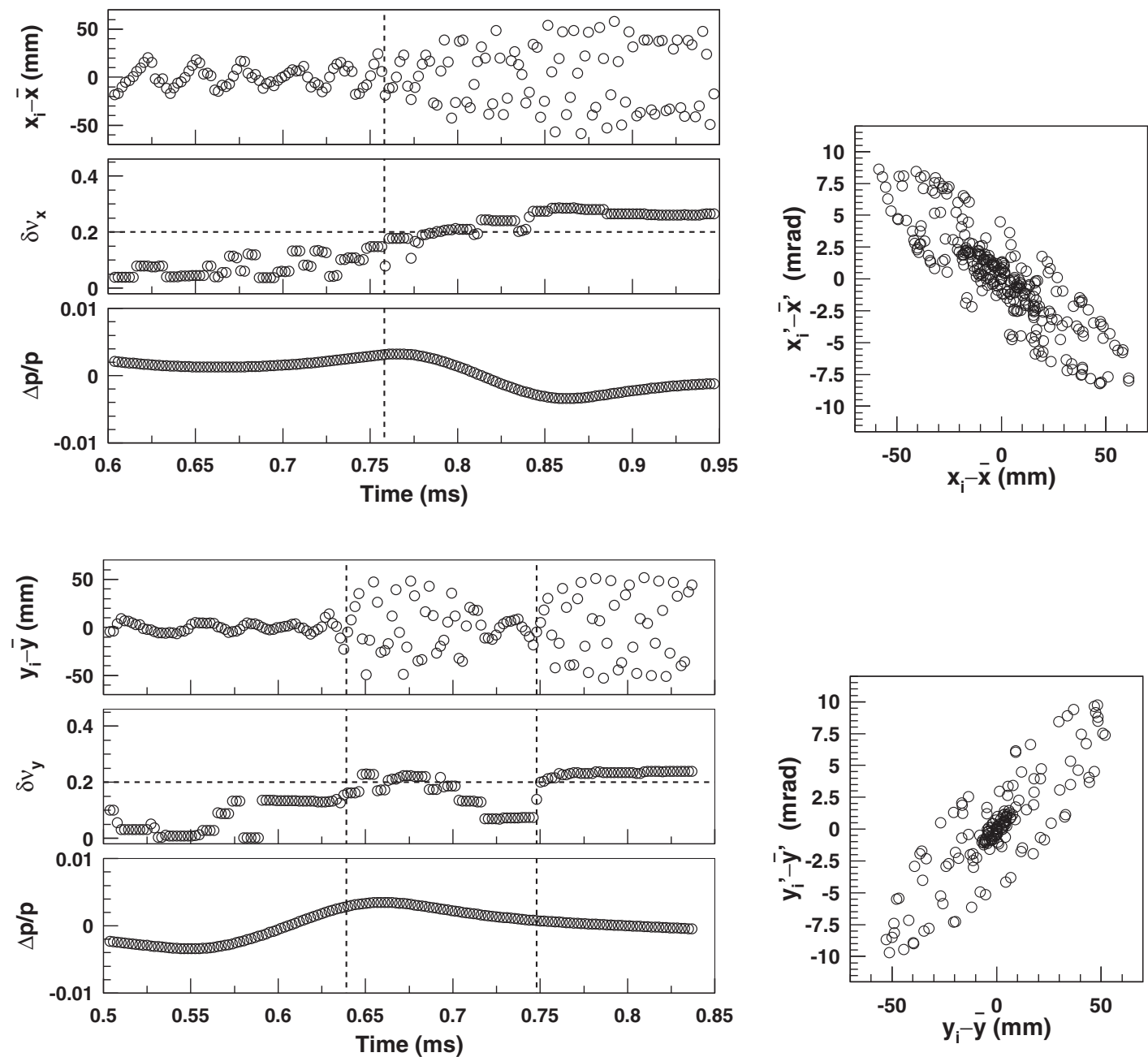

FIG. 19. Single-particle behavior of one macroparticle near $2 \delta \nu_{\chi}=0.4$ calculated for the beam intensity of $539 \mathrm{~kW}$; incoherent position $\chi_{i}-\bar{\chi}$, fractional part of incoherent tune $\delta \nu_{\chi}$, momentum deviation $\Delta p / p$, and two-dimensional transverse phase-space coordinates $\left(\chi_{i}-\bar{\chi}, \chi_{i}^{\prime}-\bar{\chi}^{\prime}\right)$. 


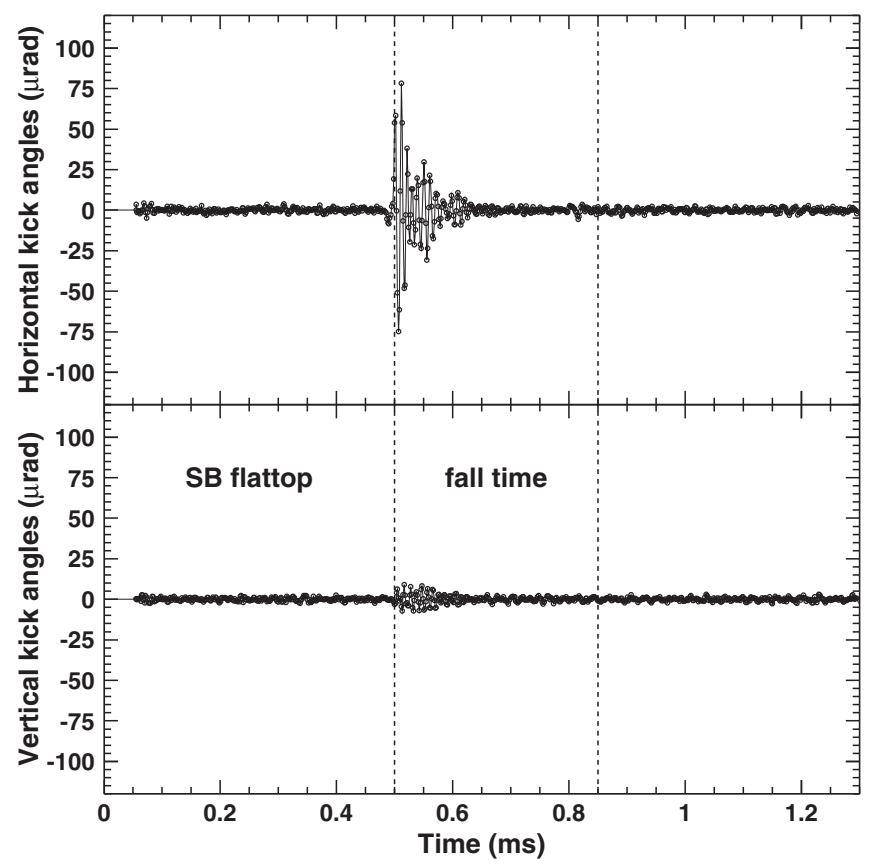

FIG. 20. Kick angles of the dipole field ripple evaluated after the improvement of the SB power supply.

halo formation and its resultant beam loss have a beam intensity dependence.

\section{Beam loss mitigation by power supply improvement}

The fundamental cause of the second bump structure of beam loss is the ripple noise of the SBs field. Therefore, it follows that the excess beam loss can be removed by mitigating the ripple noise.

With the injection energy upgrade in 2013, the SB power supply was replaced with the new one, when a new technique based on the pulse-forming system by switching capacitors was adopted to mitigate the ripple noise [22]. Thanks to the limited number of switching events in the new power supply, the ripple noise of the SBs field was well suppressed. By this improvement, the dipole field ripple affecting the beam was drastically mitigated as shown in Fig. 20. A beam test performed right after the injection energy upgrade confirmed that the second bump structure of beam loss was removed, as expected by this power supply improvement, as is shown in the right plot in Fig. 22 later.

\section{HIGH-INTENSITY BEAM TESTS OF UP TO $573 \mathrm{~kW}$ CONDUCTED AFTER INJECTION ENERGY UPGRADE}

In April and June 2014, after the injection energy upgrade, we performed high-intensity beam tests of up to $573 \mathrm{~kW}$ with the higher injection energy of $400 \mathrm{MeV}$, where the operational bare tune was set to the same point of $(6.45,6.42)$. In this beam test, we investigated the effect of the injection energy upgrade, and tried further beam loss mitigation by adjusting the injection beam Twiss parameter.

\section{A. Effect of injection energy upgrade from $181 \mathrm{MeV}$ to $400 \mathrm{MeV}$}

To verify the effect of the injection energy upgrade, we again measured the painting parameter dependence of beam loss with the higher injection energy of $400 \mathrm{MeV}$, and compared the experimental result with the previous one taken with the lower injection energy of $181 \mathrm{MeV}$.

The upper plot in Fig. 21 shows the beam survival rates measured with the systematic combinations of transverse and longitudinal painting listed in Table II. The blue circles in this figure are the previous ones evaluated from the experimental data in Fig. 10, while the red circles correspond to the new ones taken with the upgraded injection energy of $400 \mathrm{MeV}$ for a similar beam intensity of $553 \mathrm{~kW}$. As is obvious from the comparison, the beam survival rate was drastically improved after the injection energy upgrade; the beam loss for the red circles is all less than $1 \%$, and thus its parameter dependence looks to be nearly flat. By the injection energy upgrade from $181 \mathrm{MeV}$ to $400 \mathrm{MeV}$, the space-charge effect at injection is mitigated by a factor of 0.3 as per the $1 / \beta^{2} \gamma^{3}$ scaling law derived from Eq. (9). This improvement of the beam survival rate is led through such a significant space-charge mitigation.

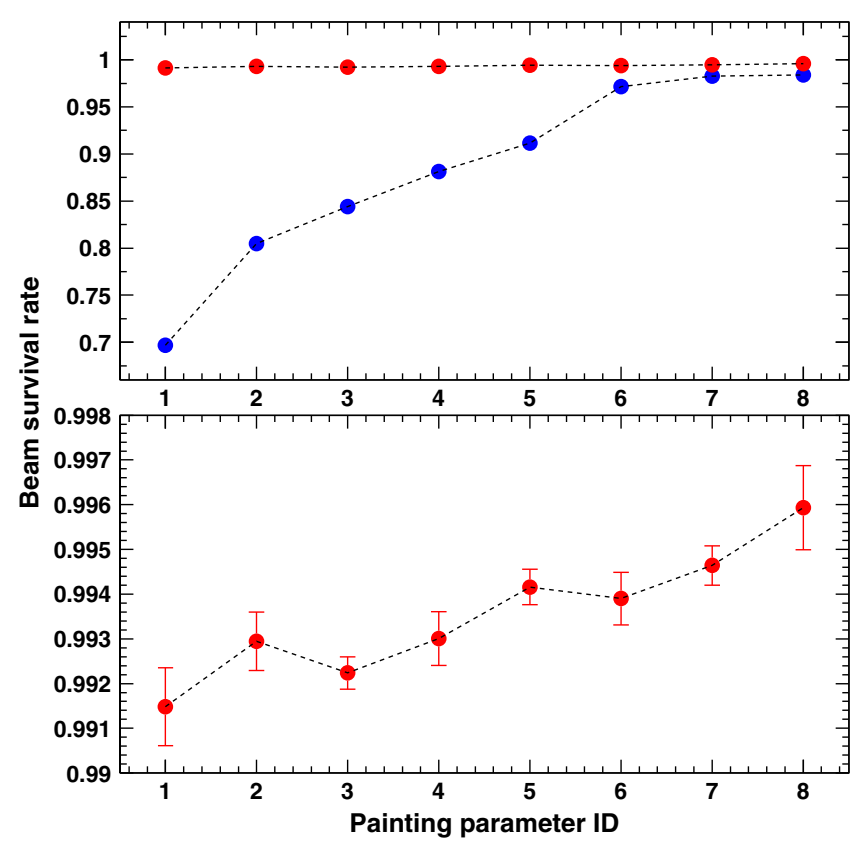

FIG. 21. (Upper) Beam survival rates measured with the systematic combinations of transverse and longitudinal painting of IDs 1-8 listed in Table II, where the blue circles are the previous values evaluated from the experimental data in Fig. 10, while the red circles correspond to the new ones taken with the upgraded injection energy of $400 \mathrm{MeV}$ for a similar beam intensity of $553 \mathrm{~kW}$. (Lower) Magnified plot of the red data in the upper plot. 

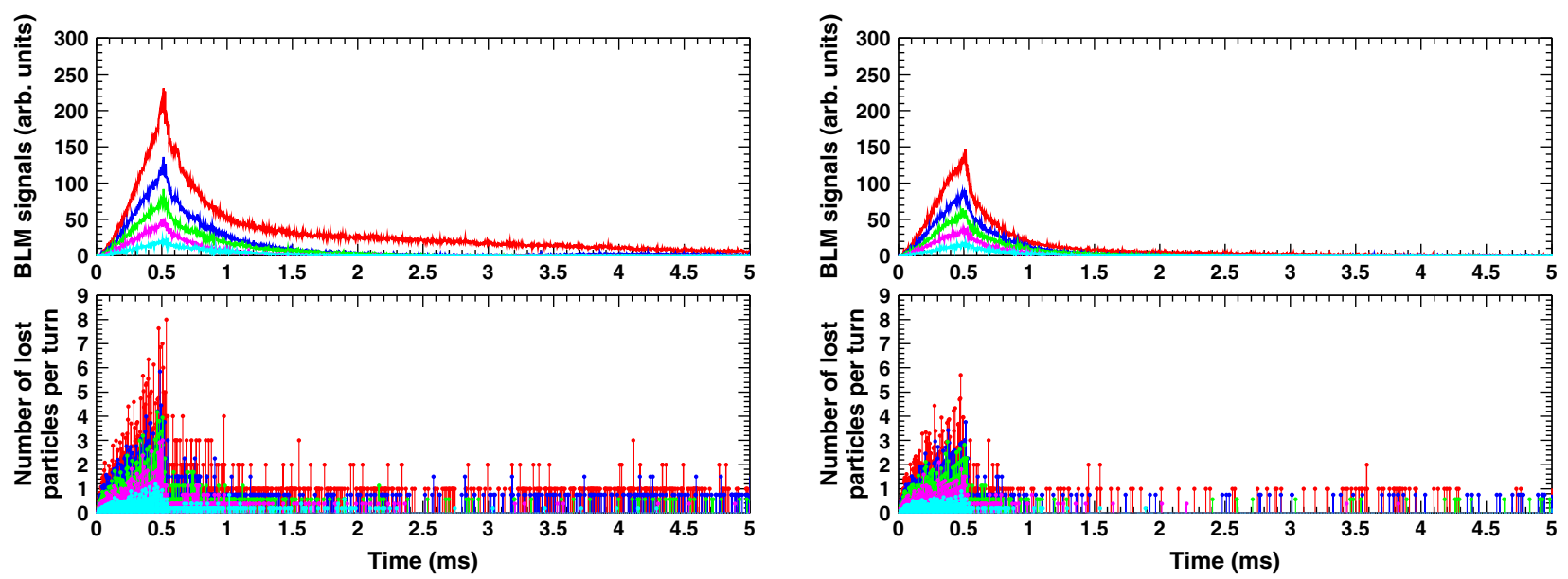

FIG. 22. (Left) Scintillation-type BLM signals over the first $5 \mathrm{~ms}$ measured at the collimator section with various beam intensities of 107 (light blue), 214 (pink), 322 (green), 429 (blue), and 573 (red) kW before the correction of the injection beam Twiss parameter (upper), and the corresponding numerical simulation results (lower). (Right) Similar results obtained after the correction of the injection beam Twiss parameter.

The lower plot in the figure shows a magnified plot of the red circles, which confirms the red circles have a similar painting parameter dependence to that of the blue ones; the beam loss was reduced from ID 1 to ID 5 by longitudinal painting, and from ID 5 to ID 8 by adding transverse painting.

These series of beam loss measurements clearly confirmed the large gain from the injection energy upgrade, as well as the excellent ability of injection painting for spacecharge mitigation.

\section{B. Beam loss caused by injection beam Twiss parameter mismatch and its mitigation}

With the injection painting parameter of ID 8, we then investigated the beam intensity dependence of beam loss to confirm the source of the residual beam loss.

The upper-left plot in Fig. 22 shows the scintillation-type BLM signals over the first $5 \mathrm{~ms}$ measured at the collimator section with various beam intensities from 107 to $573 \mathrm{~kW}$, where the beam intensity was varied by thinning the number of intermediate pulses while maintaining the injection pulse length at $0.5 \mathrm{~ms}$. As shown in the plot, the beam losses for beam intensities of up to $429 \mathrm{~kW}$ (blue) almost have similar figures and linear beam intensity dependence, but the beam loss for $573 \mathrm{~kW}$ (red) shows a different trend, rising more sharply for the first $0.5 \mathrm{~ms}$ of injection, and then trailing off more lazily. This experimental result implies that the beam loss includes an extra beam loss component other than foil scattering beam loss during charge-exchange injection.

The beam loss arising from foil scattering generally occurs in proportion to the product of the beam intensity and the foil hitting rate. In this measurement, the injection painting parameter and the injection pulse length were fixed, so the foil hitting rate during injection was almost unchanging for the present intensity variation. Thus, this experimental condition generally leads to linear beam intensity dependence regarding the foil scattering part of beam loss. That is, the excess beam loss component over linear beam intensity dependence is from sources other than foil scattering.

As shown in the lower-left plot in Fig. 22, the numerical simulation well reproduced the empirical beam loss by considering the injection beam distribution based on the measurement, and indicated that the excess beam loss originates from a Twiss parameter mismatch of the injection beam. Figure 23 shows the transverse injection painting area evaluated through measurements. In this figure, the solid ellipse represents the injection beam, while the dashed ellipse shows the painting area, which is formed by filling the injection beam ellipse along the black arrow. Here, the blue ones correspond to the case that the injection beam Twiss parameter is mismatched. One can find that the painting area is unnecessarily expanded to
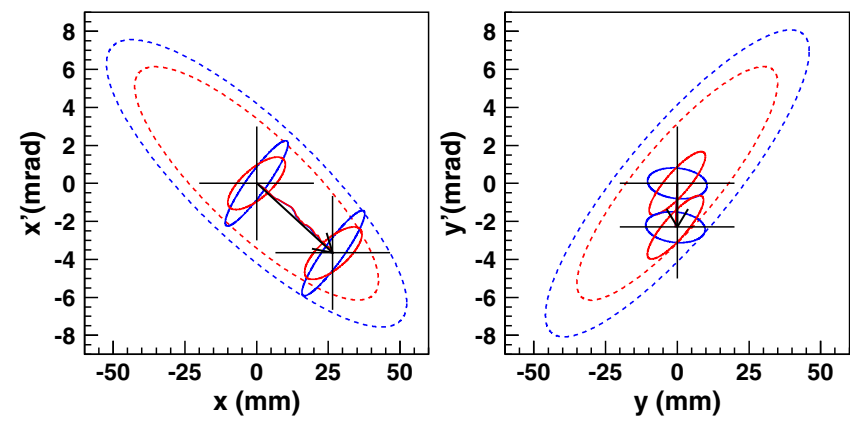

FIG. 23. Transverse injection painting areas evaluated through measurements before (blue) and after (red) the correction of the injection beam Twiss parameter; the solid ellipse represents the injection beam, while the dashed ellipse shows the painting area formed by filling the injection beam ellipse along the black arrow. 

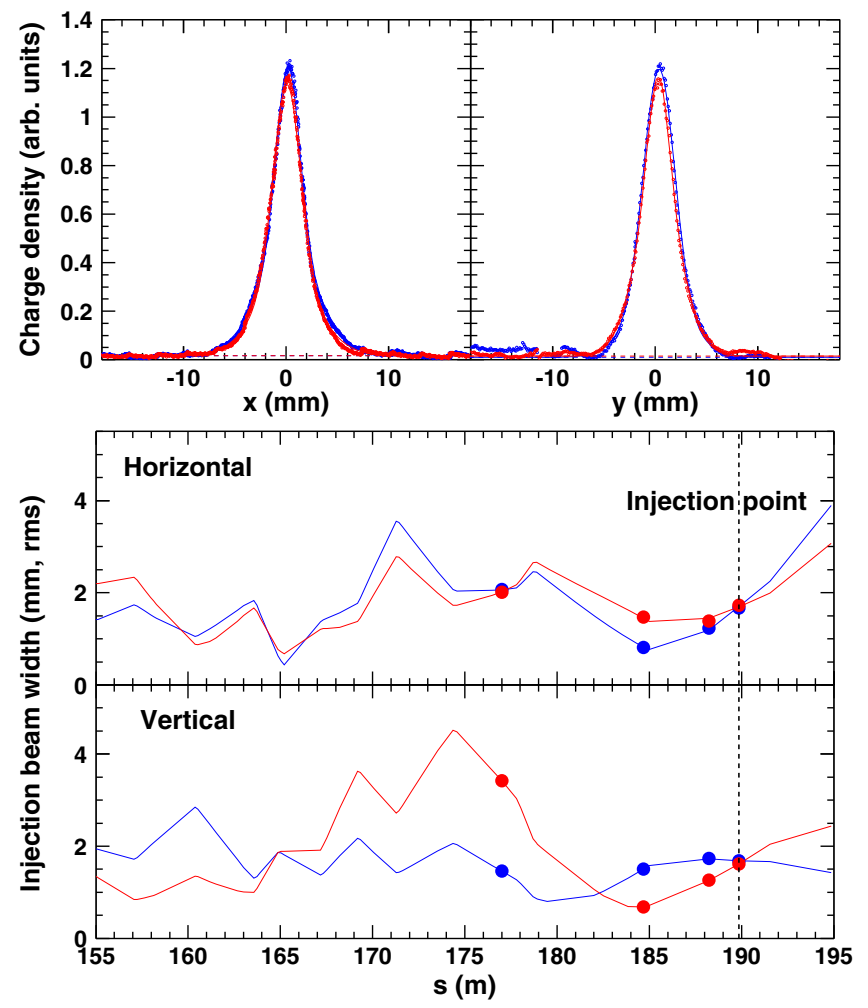

FIG. 24. (Upper) Transverse injection beam profiles measured at the RCS injection point. (Lower) Injection beam envelopes along the injection beam transport line reconstructed by model fitting to measured injection beam widths (circles). In these plots, the blue ones are those before the correction of the injection beam Twiss parameter, while the red ones are those after the correction.

$\epsilon_{t p}>160 \pi \mathrm{mm}$ mrad due to deformation of the injection beam ellipse. The numerical simulation concluded that the resultant large amplitude particles cause the observed excess beam loss. This error in the injection beam Twiss parameter occurred unexpectedly with the injection energy upgrade.

Based on the above consideration, we attempted to mitigate the excess beam loss component. The upper plot in Fig. 24 shows the injection beam profiles measured at the RCS injection point, while the lower plot in Fig. 24 shows the injection beam envelopes along the injection beam transport line reconstructed by model fitting to the measured injection beam profiles. The injection beam Twiss parameter at the RCS injection point was evaluated from the beam envelope analysis, and then corrected by using several sets of quadrupole magnets in the injection beam transport line [23]. By this adjustment, the painting area was well modified to be $\epsilon_{t p}=100 \pi \mathrm{mm}$ mrad, as shown in the red plot in Fig. 23. As a result of this effort, the excess beam loss was successfully mitigated as predicted by the numerical simulation, as shown in the right plots in Fig. 22.

The beam loss after the correction occurs only for the first $1 \mathrm{~ms}$ when the injection bump is activated, and its amount generally shows linear beam intensity dependence to the end. The experimental results state that the residual beam loss estimated to be $0.1 \%$ is mainly from foil scattering during injection, and that the other beam losses are nearly minimized for beam intensities of up to $573 \mathrm{~kW}$.

\section{1-MW BEAM TESTS CONDUCTED AFTER INJECTION CURRENT UPGRADE}

In October 2014, right after the injection current upgrade, we launched a 1-MW high-intensity beam test using a $0.5 \mathrm{~ms}$-long injection pulse with a peak current of $45.0 \mathrm{~mA}$ and a chopper beam-on duty factor of $60 \%$, where the number of particles per pulse was $8.45 \times 10^{13}$.

In order to survey the high-intensity aspects of such a MW-class high-power beam operation, we first performed various beam dynamics measurements using the operational parameters optimized by then for beam intensities of up to $600 \mathrm{~kW}$; the bare tune was set to $(6.45,6.42)$ and the injection painting of ID 8 was used.

\section{A. Longitudinal beam loss and its mitigation}

The first 1-MW beam test was performed in October 2014. In this trial, beam acceleration of up to $773 \mathrm{~kW}$ was achieved with no significant beam loss, but 1-MW beam acceleration was not reached due to over current in the rf anode power supply. Since a higher-intensity beam needs larger beam loading compensation, the workload of the rf power supply increases with beam intensity ramp-up.

After this beam test, a quick measure against the rf trip was taken; the resonant frequency of the rf cavity was shifted from 1.7 to $2.1 \mathrm{MHz}$ to decrease the anode current required for the 1-MW beam acceleration. By this treatment, the 1-MW beam acceleration was successfully achieved in January 2015. But, at this stage, there still remained slight longitudinal beam loss $\left(<10^{-3}\right)$ due to a rf bucket distortion caused by beam loading. Such a longitudinal beam loss occurs through a large momentum excursion, so it leads to unlocalized beam loss at the arc section with high dispersion. The upper plot in Fig. 25 shows the scintillation-type BLM signals over the whole acceleration time of $20 \mathrm{~ms}$ measured at the arc section with various beam intensities of up to $1 \mathrm{MW}$. As shown in the figure, a significant beam loss signal is not detected up to the beam intensity of $825 \mathrm{~kW}$, but appearing when the beam intensity goes beyond $900 \mathrm{~kW}$ (blue and red).

RCS employs a multiharmonic feed-forward (FF) system for beam loading compensation [24], and it works very well. But, the rf anode power supply nearly reached the limit again at this stage, and there remained not enough margin to achieve adequate beam loading compensation for the beam intensity of $1 \mathrm{MW}$. This is the fundamental cause of the longitudinal beam loss.

The resonant frequency shift is also another possible cause of the beam loss. RCS normally accelerates two 


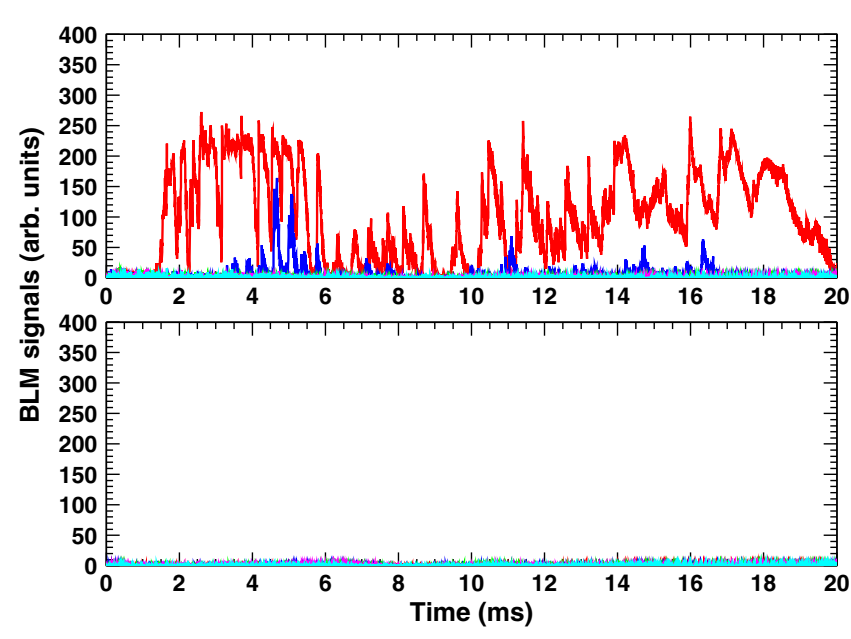

FIG. 25. Scintillation-type BLM signals over the whole time of $20 \mathrm{~ms}$ measured at the arc section with various beam intensities of 568 (light blue), 696 (pink), 825 (green), 944 (blue) kW and $1 \mathrm{MW}$ (red) before (upper) and after (lower) the rf power supply upgrade.

bunches with the harmonic number of $h=2$. Thus, major parts of wake voltage components are even harmonics ( $h=2,4$, and 6). But, after the resonant frequency shift, the effect of odd harmonics ( $h=1,3$, and 5) was additionally enhanced [25], causing different longitudinal motions for the two bunches, as shown in the upper plot in Fig. 26. Our FF system covers the odd harmonics [26] as well as the even ones, but the phenomenon made the FF tuning more complicated.

Receiving this result, the rf anode power supply was upgraded in the scheduled summer maintenance period in 2015. Then, the resonant frequency of the rf cavity was restored to the original value. By these hardware improvements and the subsequent beam loading compensation, stable longitudinal motion was established, as seen in the lower plot in Fig. 26. As a result of this effort, the longitudinal beam loss was completely eliminated even for the beam intensity of $1 \mathrm{MW}$, as shown in the lower plot in Fig. 25.

\section{B. Transverse beam loss and its localization}

The 1-MW beam test after the rf power supply upgrade confirmed that most of remaining transverse beam loss is well localized at the collimator section in the dispersionfree long straight insertion.

Figure 27 shows the scintillation-type BLM signals for the first $5 \mathrm{~ms}$ measured at the collimator section with various beam intensities of up to $1 \mathrm{MW}$. Similarly to the right plot in Fig. 22, the beam loss occurs only for the first $1 \mathrm{~ms}$, and its amount nearly shows linear response with the product of the beam intensity and the foil hitting rate. As discussed in the last section, the experimental result means that the observed beam loss mainly arises from foil scattering during charge-exchange injection. The other

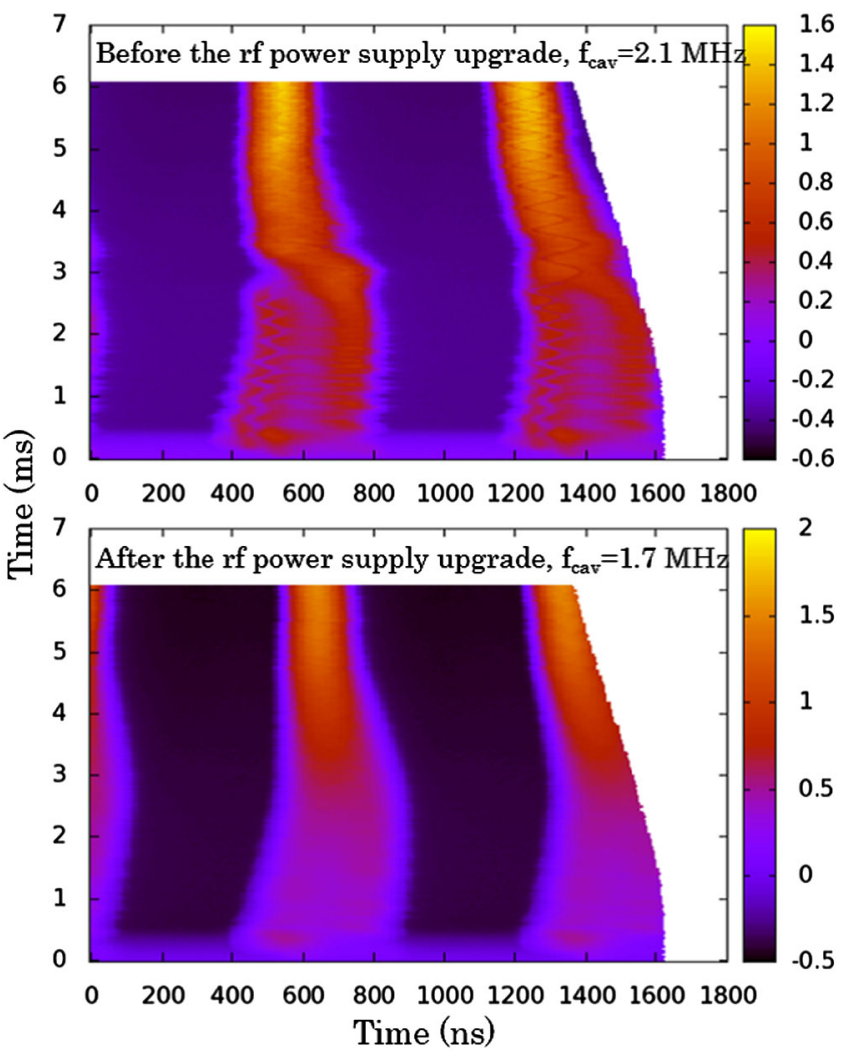

FIG. 26. Mountain plots of the wall current monitor measured for the beam intensity of $1 \mathrm{MW}$ before (upper) and after (lower) the rf power supply upgrade.

beam loss, such as space-charge induced beam loss, was well minimized even for the beam intensity of $1 \mathrm{MW}$ by the combination of transverse and longitudinal painting of ID 8 .

The beam loss observed for the beam intensity of $1 \mathrm{MW}$ was estimated to be $0.1 \%$. This beam loss rate corresponds to power of $133 \mathrm{~W}$, which is considerably lower than the collimator's capability of $4 \mathrm{~kW}$.

\section{Beam instability and its suppression}

Beam instability was also an important issue for the 1-MW beam acceleration. In RCS, the extraction pulse

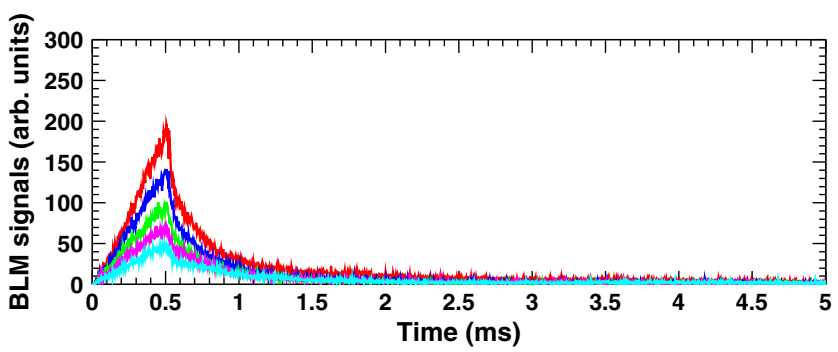

FIG. 27. Scintillation-type BLM signals over the first $5 \mathrm{~ms}$ measured at the collimator section with various beam intensities of 473 (light blue), 606 (pink), 731 (green), 870 (blue) kW and $1 \mathrm{MW}$ (red) after the rf power supply upgrade. 


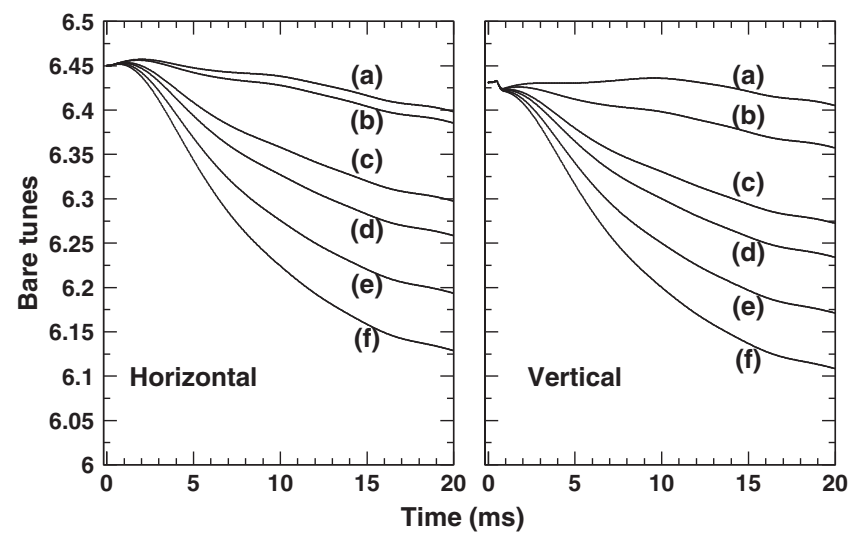

FIG. 28. Six sets of tune variations during acceleration applied for the beam instability measurement.

kicker is the most dominant impedance source, causing horizontal beam instability depending on the choice of operational parameters such as betatron tune and chromaticity $[27,28]$. Thus, for its suppression, systematic beam instability measurement was conducted with different tunes and chromaticities in the initial stage of the 1-MW beam test.

Figure 28 shows six sets of tune variations from injection to extraction used for this measurement; the tunes at injection were set at the same point of (6.45, 6.42 ), but thereafter, they were moved differently toward extraction by controlling the main quadrupole field patterns. Figure 29 shows the time dependence of the turn-by-turn horizontal beam position, measured with the six sets of tune variations (a)-(f) for the beam intensity of $1 \mathrm{MW}$. The blue plots in this figure show the case in which the natural chromaticity $\left(\xi_{x, y} \approx-9\right)$ is fully corrected to zero at injection with dc sextupole fields. In this case, beam instability occurs for any tune variation, whereas the growth rate displays a characteristic tune dependence, which is mainly determined by the frequency dependence of the kicker impedance. On the other hand, the green and the red plots correspond to the cases of less chromaticity correction; only a quarter of the natural chromaticity is corrected at injection with dc sextupole fields in case of the green ones, while no chromaticity correction is applied to the red ones. In this figure, one can find the beam instability is more stabilized by Landau damping through momentum spreads as the negative chromaticity increases. This situation allows us to fully suppress beam instability even for the beam intensity of $1 \mathrm{MW}$ in combination with dynamical tune control during acceleration.

The operational condition to damp beam instability was revealed through this measurement. Figures 25 and 27, presented above, are the experimental data taken under the stable condition, that is, with the combination of tune variation (d) and less chromaticity correction (green), where no instability occurs.

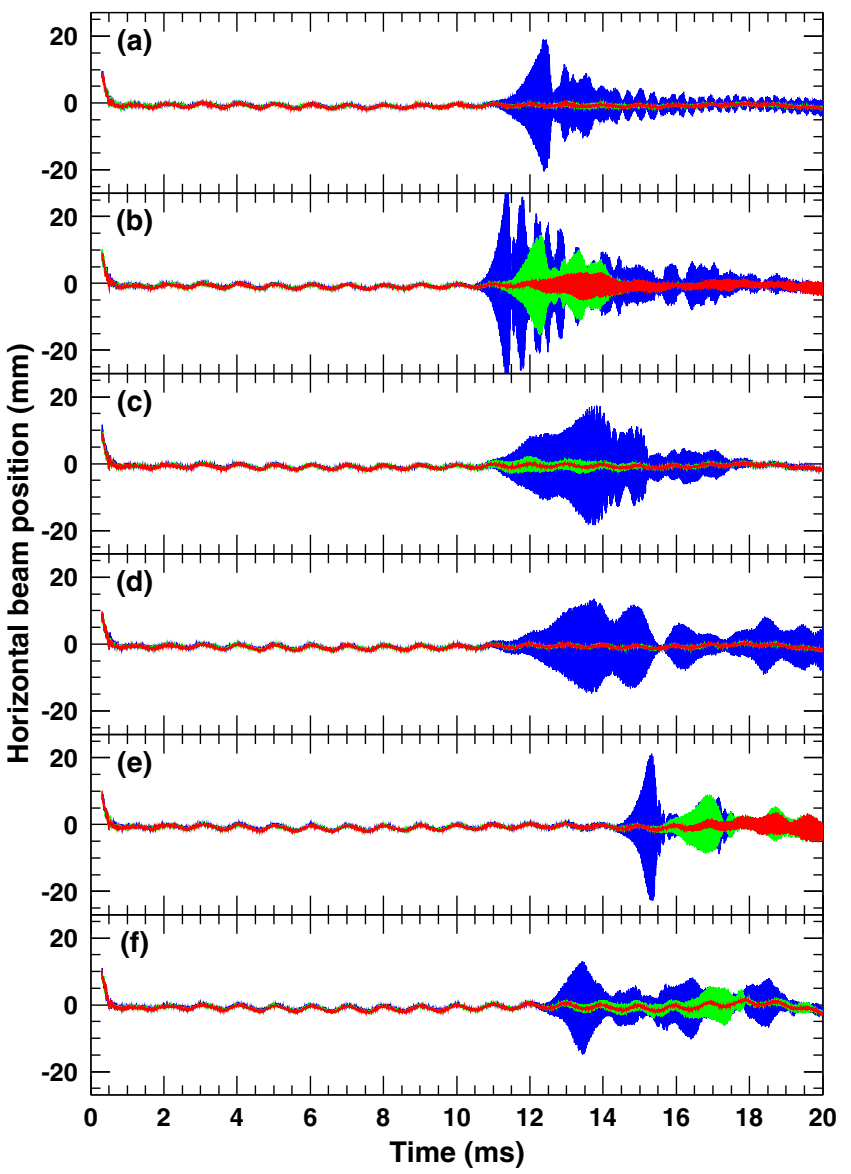

FIG. 29. Horizontal beam positions over the whole time of $20 \mathrm{~ms}$ measured for the beam intensity of $1 \mathrm{MW}$ with the six sets of tune variations (a)-(f) given in Fig. 28, where the color variations show differences in the degree of chromaticity correction; (blue) the natural chromaticity is fully corrected to zero at injection with dc sextupole fields, (green) only a quarter of the natural chromaticity is corrected at injection with dc sextupole fields, and (red) no chromaticity correction is applied.

\section{FURTHER BEAM LOSS MITIGATION BY WIDER TRANSVERSE INJECTION PAINTING}

As described in the last section, beam loss other than foil scattering beam loss is well minimized for beam intensities of up to $1 \mathrm{MW}$. Thus, the next assignment is to further reduce the foil scattering beam loss.

Most of the foil scattering beam loss is well localized at the collimator section, so no serious problem has been encountered to date. But some particles with large scattering angles cause unlocalized beam loss, leading to relatively high machine activation near the charge-exchange foil. The residual dose level there is predicted to reach several $10 \mathrm{mSv} / \mathrm{h}$ on the chamber surface if the output beam power is increased to $1 \mathrm{MW}$ as is. Many devices in the injection section often need maintenance. Thus, the residual dose level has to be reduced as low as possible to ensure a better hands-on-maintenance environment. 
The amount of foil scattering beam loss is in proportion to the foil hitting rate during injection. One possible solution to reduce the foil hitting rate is to expand the transverse painting area. As shown in Fig 4, horizontal painting is performed by a horizontal closed orbit variation during injection. Thus, the foil hitting rate decreases as the horizontal painting area becomes wider, because the circulating beam more rapidly escapes from the foil thanks to the larger horizontal closed orbit variation. On the other hand, vertical painting is performed by a vertical injection angle change during injection. The foil hitting rate can be reduced also by vertical painting by introducing the wider painting area than the vertical dimension of the foil. The painting emittance used thus far is $\epsilon_{t p}=100 \pi \mathrm{mm} \mathrm{mrad}$, which is adequate for spacecharge mitigation. But, in this case, the average number of foil hits per particle is as large as 20.0. This number can be reduced to 11.1 or 5.4 if the painting emittance is expanded to $\epsilon_{t p}=150 \pi$ or $200 \pi \mathrm{mm} \mathrm{mrad}$.

This section describes our recent efforts toward expanding the transverse painting area.

\section{A. Expanding transverse painting area to $\epsilon_{t p}=150 \pi \mathrm{mm} \mathrm{mrad}$}

Wide-ranging transverse injection painting with $\epsilon_{t p}>$ $100 \pi \mathrm{mm}$ mrad had not been realized until recently due to edge focus which occurs at the entrance and the exit of pulsed injection bump magnets (SBs and PBHs) when the injection bump is activated for injection. As shown in the left plot in Fig. 30, the edge focus causes a 30\% considerable betatron amplitude function beating on the vertical plane during injection. This betatron amplitude function beating breaks the lattice super-periodicity, and additionally excites random betatron resonances. Such random

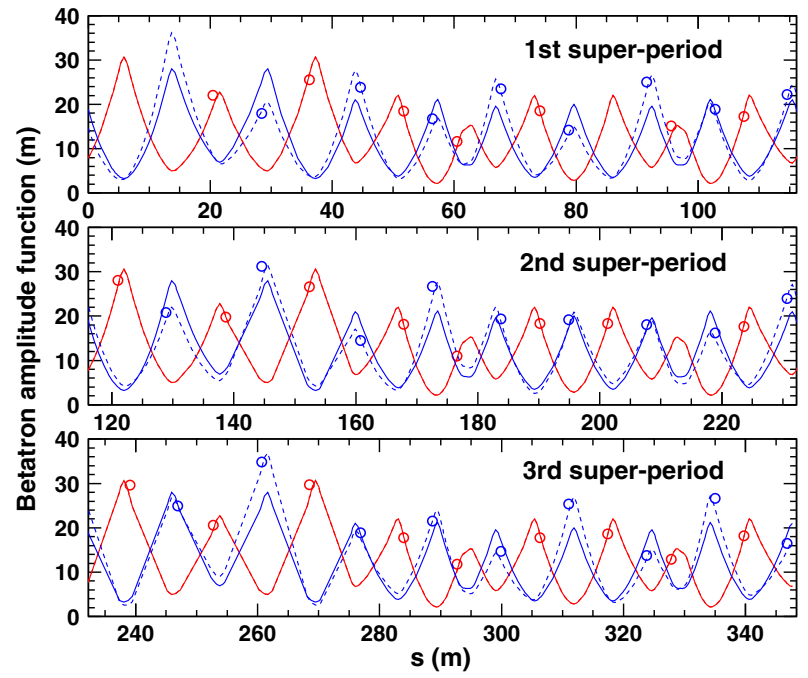

resonances lead to shrinkage of the dynamic aperture during the injection period, causing significant extra beam loss when the transverse painting area is enlarged.

Efforts to solve this issue has been reported in Ref. [29], but we have included the brief summary here for the convenience of explaining subsequent works in the next subsection.

\section{Beam loss mitigation by introducing quadrupole correctors}

As shown in Fig. 1, we recently installed six sets of pulse type quadrupole correctors (QDT1 - 6) to compensate the betatron amplitude function beating during injection [30]. By introducing QDTs, the betatron amplitude function beating was well corrected to less than $5 \%$, as shown in the right plot in Fig. 30. The effect of the random betatron resonances can be minimized through the recovery of the superperiodic condition.

In order to confirm the effectiveness of this correction scheme, we conducted a beam test with the beam intensity of $850 \mathrm{~kW}$. While the operational point used so far was $(6.45,6.42)$, it was slightly changed to $(6.45,6.38)$ in this beam test. The reason for this modification is revealed later. The left plot in Fig. 31 shows the experimental result. As shown in the plot, $0.4 \%$-extra beam loss occurred when the transverse painting area was enlarged from $100 \pi$ to $150 \pi \mathrm{mm} \mathrm{mrad}$, but the beam loss was well minimized as expected by introducing QDTs.

\section{Discussion for beam loss and its mitigation mechanisms}

As shown in the right plot in Fig. 31, the empirical result was well reproduced by the numerical simulation. With this

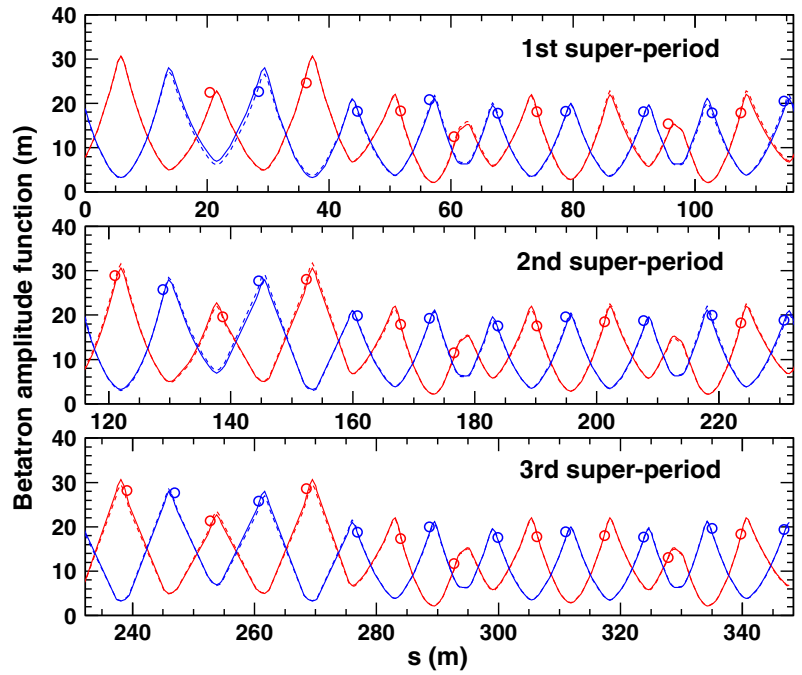

FIG. 30. Horizontal (red circles) and vertical (blue circles) betatron amplitude functions measured without (left) and with (right) QDTs when the injection bump is active, where the dashed curves are the corresponding calculated results, while the solid ones are the unmodulated betatron amplitude functions calculated with no injection bump. 

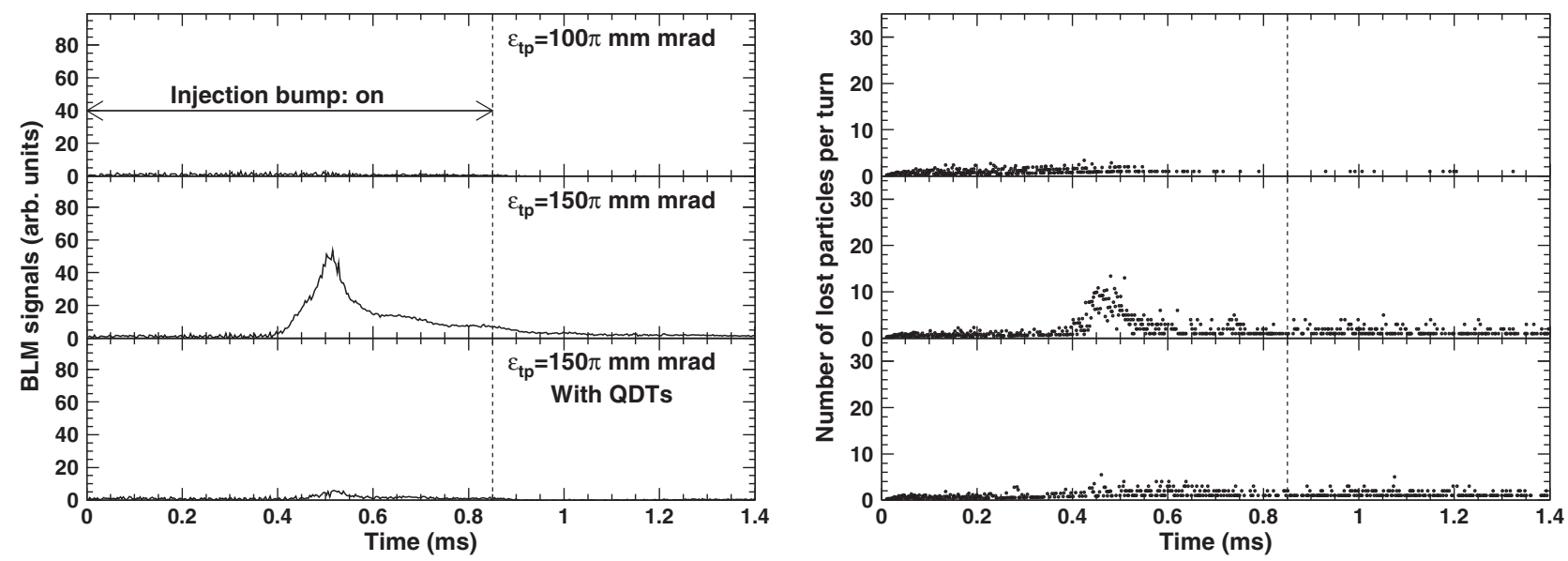

FIG. 31. (Left) Scintillation-type BLM signals over the first $1.4 \mathrm{~ms}$ measured at the collimator section with the beam intensity of $850 \mathrm{~kW}$; (top) $\epsilon_{t p}=100 \pi \mathrm{mm} \mathrm{mrad}$, (middle) $\epsilon_{t p}=150 \pi \mathrm{mm} \mathrm{mrad}$, and (bottom) $\epsilon_{t p}=150 \pi \mathrm{mm} \mathrm{mrad} \mathrm{with} \mathrm{QDTs.} \mathrm{(Right)}$ Corresponding numerical simulation results.

numerical simulation result, the mechanism of the observed phenomena was investigated in more detail.

Figure 32 shows the transverse phase space coordinates calculated at the end of injection with $\epsilon_{t p}=$ $150 \pi \mathrm{mm} \mathrm{mrad}$, where three kinds of transverse beam distributions are given; (a) without edge focus, (b) with edge focus, and (c) with the addition of QDTs to (b). In this figure, one can find that beam halo formation is enhanced from (a) to (b) due to the edge focus, especially on the vertical plane. This beam halo formation causes the extra beam loss observed in the middle plot in Fig. 31. But, the beam halo is well mitigated from (b) to (c) by QDTs, which results in the beam loss reduction observed in the bottom plot in Fig. 31.

Figure 33 shows the tune diagram near the present operating point. The numerical simulation confirmed that the beam halo is formed by the combined effect of the following two resonances;

$$
\begin{aligned}
& \nu_{x}+2 \nu_{y}=19, \\
& 2 \nu_{x}-2 \nu_{y}=0 .
\end{aligned}
$$

The $\nu_{x}+2 \nu_{y}=19$ resonance is a third-order random resonance arising from the chromatic correction sextupole field and the intrinsic sextupole field component in the main bending magnets, and it is additionally excited through a distortion of the superperiodicity caused by the edge focus during injection. This nonlinear sum resonance induces emittance growth on both the horizontal and the vertical planes with the invariant value of $2 J_{x}-J_{y}$ [31], where $J_{x}$ and $J_{y}$ are the horizontal and the vertical betatron actions. Specifically, this resonance leads to two times larger emittance growth on the vertical plane than that on the horizontal plane. On the other hand, the $2 \nu_{x}-2 \nu_{y}=0$ resonance is a fourth-order systematic resonance, which is mainly excited by the octupole component in the space-charge field. Therefore, it affects the beam regardless of the edge focus. This nonlinear difference resonance induces emittance exchange between the horizontal and the vertical planes with the invariant value of $J_{x}+J_{y}[32,33]$.

Figure 34 shows a two-dimensional plot of the turn-byturn betatron actions $\left(J_{x}, J_{y}\right)$ of one macroparticle which results in the beam halo found in Fig. 32(b). In this figure, one can see a characteristic emittance blow-up that implies the combined effect of the two resonances; the horizontal
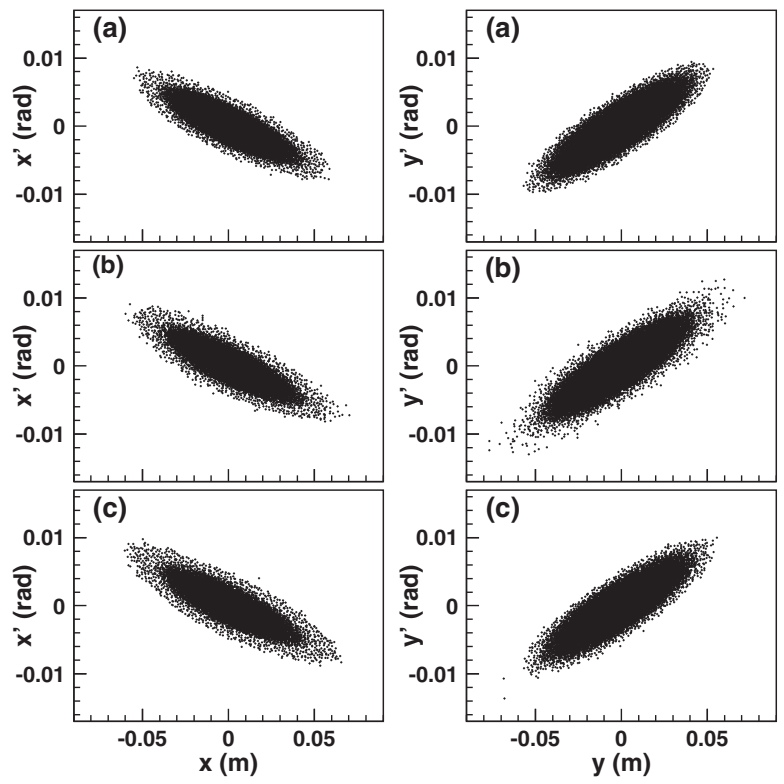

FIG. 32. Two-dimensional transverse phase space coordinates at the end of injection calculated with $\epsilon_{t p}=150 \pi \mathrm{mm} \mathrm{mrad}$ for the beam intensity of $850 \mathrm{~kW}$, where three kinds of transverse beam distributions are given; (a) without edge focus, (b) with edge focus, and (c) with the addition of QDTs to (b). 


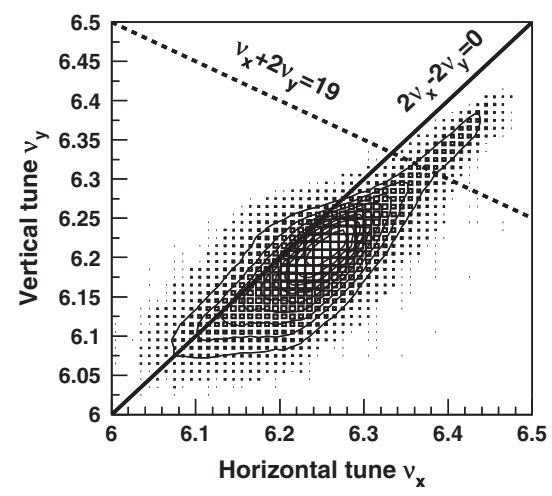

FIG. 33. Tune footprint at the end of injection calculated with $\epsilon_{t p}=150 \pi \mathrm{mm}$ mrad for the beam intensity of $850 \mathrm{~kW}$.

and the vertical actions of the macroparticle gradually grow up along the line of $2 J_{x}-J_{y}=$ const, while oscillating in the direction parallel to the line of $J_{x}+J_{y}=$ const. This analysis confirmed that most of the beam halo is generated through such a single-particle behavior. In particular, the contribution of the $\nu_{x}+2 \nu_{y}=19$ resonance is more critical, because it leads to more severe beam halo formation on the vertical plane. QDTs act to mitigate the $\nu_{x}+$ $2 \nu_{y}=19$ resonance through the recovery of the superperiodic condition. Hence it follows that the beam loss reduction achieved in this beam test is mainly led by QDTs through the mitigation of the vertical beam halo.

By these series of beam experiments and numerical simulations, the transverse painting area was successfully expanded to $\epsilon_{t p}=150 \pi \mathrm{mm}$ mrad with no significant

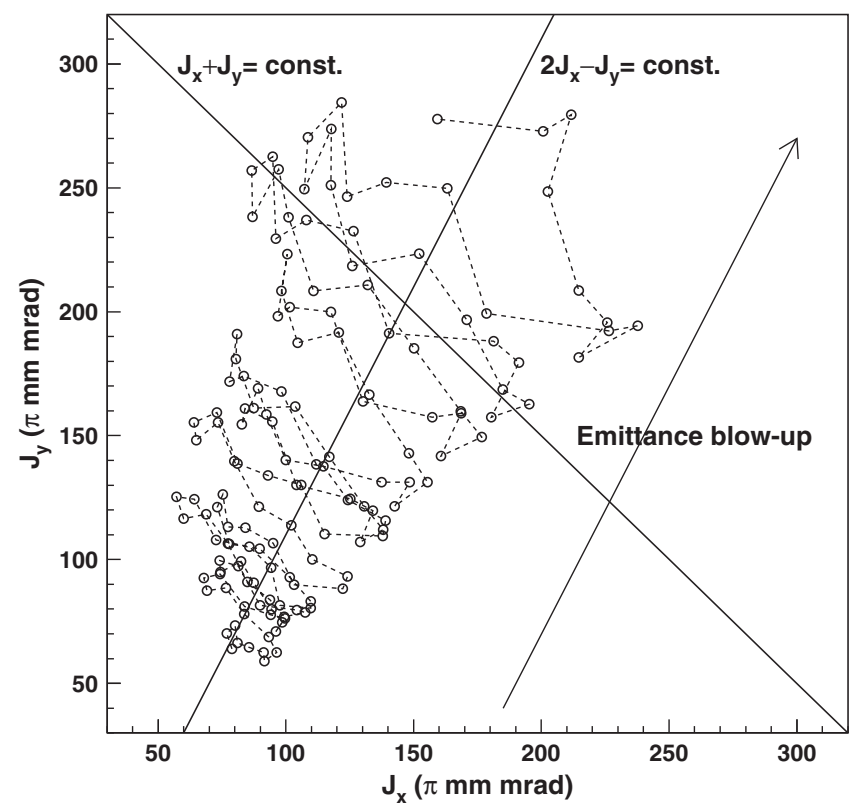

FIG. 34. Typical sample of the incoherent motion of one macroparticle which results in the beam halo found in Fig. 32(b); twodimensional plot of the tune-by-turn betatron actions $\left(J_{x}, J_{y}\right)$. additional beam loss. After this successful beam tuning, the routine output beam power for the user program was increased to $500 \mathrm{~kW}$.

\section{B. Expanding transverse painting area to $\epsilon_{t p}=200 \pi \mathrm{mm} \mathrm{mrad}$}

The above analysis provided us with another important guideline for beam tuning. That is, it suggested that further expansion of the transverse painting area can be realized by alleviating the effect of the $2 \nu_{x}-2 \nu_{y}=0$ resonance, as well as mitigating the $\nu_{x}+2 \nu_{y}=19$ resonance with QDTs. As mentioned in the last subsection, the operational point was slightly changed from $(6.45,6.42)$ to $(6.45,6.38)$ when we launched a campaign for expanding the transverse painting area. This modification acts to alleviate the effect of the $2 \nu_{x}-2 \nu_{y}=0$ resonance, but the further mitigation is required for realizing wider transverse injection painting such as $\epsilon_{t p}=200 \pi \mathrm{mm}$ mrad. To address this issue, we discussed the introduction of anticorrelated painting as a substitute for correlated painting used thus far.

\section{Anticorrelated painting vs. correlated painting}

In RCS, anticorrelated painting [14,15] as well as correlated painting are available. As mentioned in Sec. IV, in correlated painting used so far, the injection beam is filled from the middle to the outside on both the horizontal and the vertical planes following the combination of Eqs. (6) and (7). On the other hand, in anticorrelated painting, the direction of vertical painting is reversed by changing the excitation waveform of PBVs; the vertical injection angle varies from the maximum angle to 0 degrees as follows;

$$
y_{\text {paint }}=0, \quad y_{\text {paint }}^{\prime}=-y_{\max }^{\prime} \sqrt{1-t / T} .
$$

That is, in anticorrelated painting, the injection beam is filled from the middle to the outside on the horizontal plane, while it is painted from the outside to the middle on the vertical plane, as per the combination of Eqs. (6) and (17).

Figure 35 shows the transverse phase space coordinates and the tune footprints at $0.16 \mathrm{~ms}$ (the first one-third of injection) and $0.5 \mathrm{~ms}$ (the end of injection) calculated for the beam intensity of $1 \mathrm{MW}$ with correlated and anticorrelated painting of $\epsilon_{t p}=200 \pi \mathrm{mm} \mathrm{mrad}$. As shown in the figure, correlated and anticorrelated painting generate different space-charge detuning in the beam injection process. In correlated painting, the charge density per beam emittance is nearly unchanging throughout the painting process. Consequently, the tune footprint is almost kept constant during injection, as shown in the upper plots. On the other hand, in anticorrelated painting, the balance of charge densities on the horizontal and the vertical planes varies during injection, so both the magnitude and the direction of the tune shift dynamically change with the 

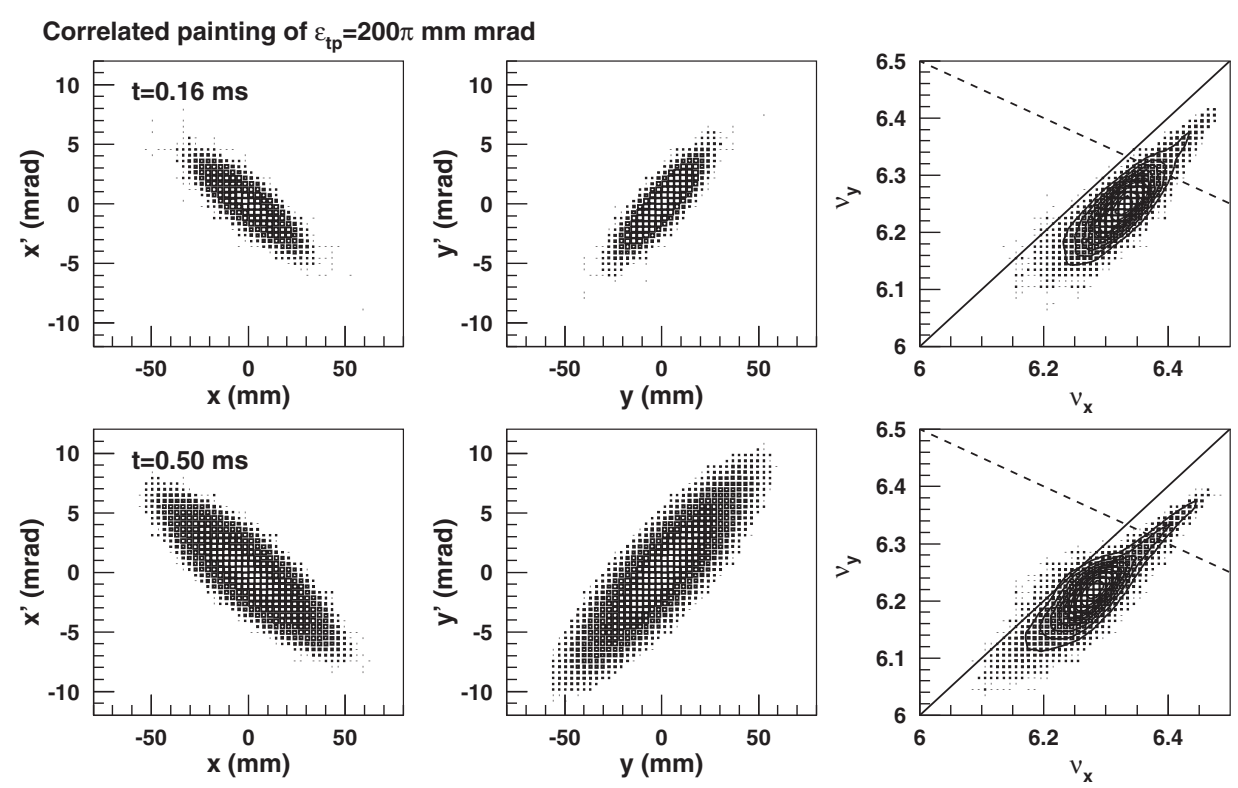

Anti-correlated painting of $\varepsilon_{\mathrm{tp}}=200 \pi \mathrm{mm} \mathrm{mrad}$
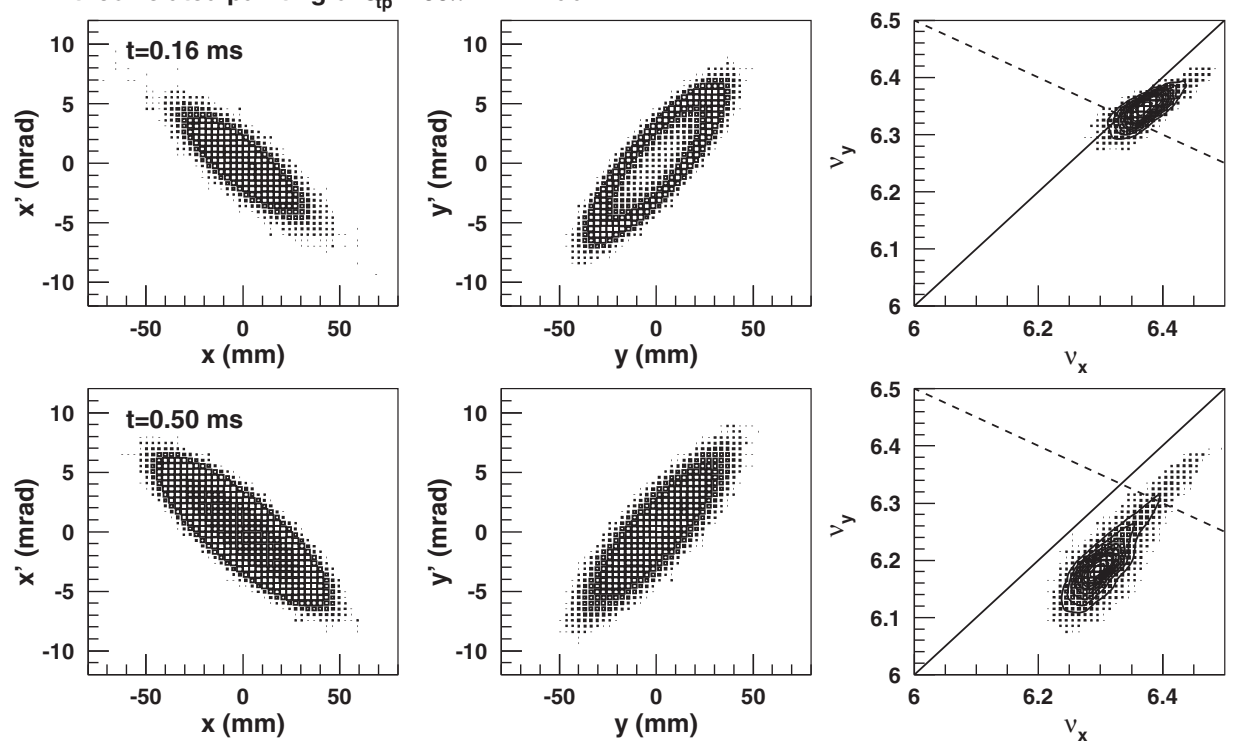

FIG. 35. Transverse phase space coordinates and tune footprints at $0.16 \mathrm{~ms}$ (the first one-third of injection) and $0.5 \mathrm{~ms}$ (the end of injection) calculated for the beam intensity of $1 \mathrm{MW}$ with correlated (upper) and anticorrelated (lower) painting of $\epsilon_{t p}=200 \pi \mathrm{mm} \mathrm{mrad}$.

progression of injection painting, as shown in the lower plots.

In addition, they display a remarkable difference in terms of the influence of the $2 \nu_{x}-2 \nu_{y}=0$ resonance. Figure 36 shows two-dimensional plots of $\left(J_{x}, J_{y}\right)$ at the end of injection calculated for the beam intensity of 1 MW with correlated and anticorrelated painting of $\epsilon_{t p}=200 \pi \mathrm{mmmrad}$. As shown in the left plot, in correlated painting, the injection beam is painted along $J_{x}-J_{y} \approx 0$, as shown by the light blue arrow. To this direction of beam painting, the emittance exchange caused by the $2 \nu_{x}-2 \nu_{y}=0$ resonance occurs in the orthogonal direction, as shown by the red arrow. Thus, the emittance exchange is directly connected to emittance growth in this case; one can find a lot of beam particles out on the painting area indicated by the dashed light-blue lines. On the other hand, in anticorrelated painting, the injection beam is painted along $J_{x}+J_{y} \approx$ const, as shown in the right plot. This direction of beam painting is the same as that of emittance exchange. Therefore, the additional emittance growth caused by the emittance exchange is well suppressed in this case. This characteristic of anticorrelated painting favors the expansion of the transverse painting area.

Another feature of anticorrelated painting is to form a Kapchinsky-Vladimirsky (KV)-like distribution. As is well 

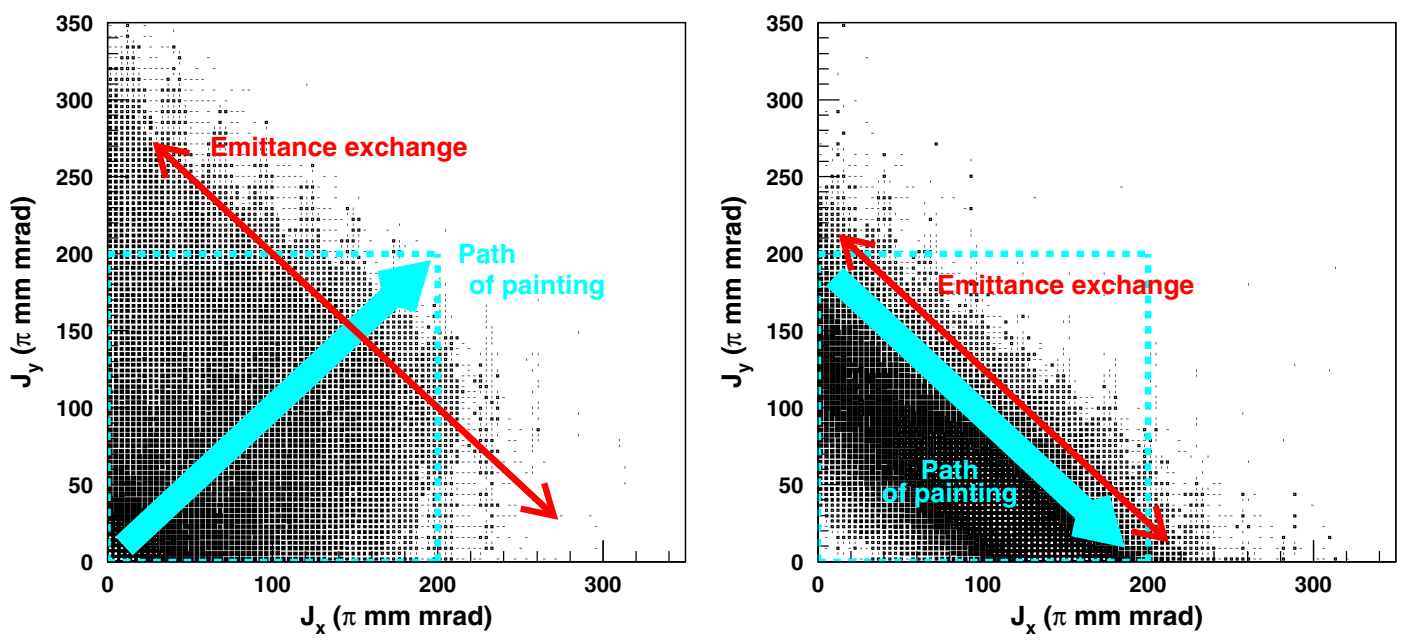

FIG. 36. Two-dimensional plots of $\left(J_{x}, J_{y}\right)$ at the end of injection calculated for the beam intensity of 1 MW with correlated (left) and anticorrelated (right) painting of $\epsilon_{t p}=200 \pi \mathrm{mm}$ mrad.

known, the KV distribution [34], where the particles are uniformly distributed strictly along the line of $J_{x}+J_{y}=$ const, only generates a linear defocusing field without producing any nonlinear field components. Because anticorrelated painting works as the analogy, it serves to reduce nonlinear components in the space-charge field. As shown in Fig. 35, anticorrelated painting generates narrower incoherent tune spread at the end of injection, compared to the case of correlated painting. This means that anticorrelated painting leads to less octupole spacecharge field than does correlated painting. Since the main source of the $2 \nu_{x}-2 \nu_{y}=0$ resonance is the octupole component, anticorrelated painting more favors the mitigation of the $2 \nu_{x}-2 \nu_{y}=0$ resonance.

Thus, anticorrelated painting has several advantages in mitigating the effect of the $2 \nu_{x}-2 \nu_{y}=0$ resonance.

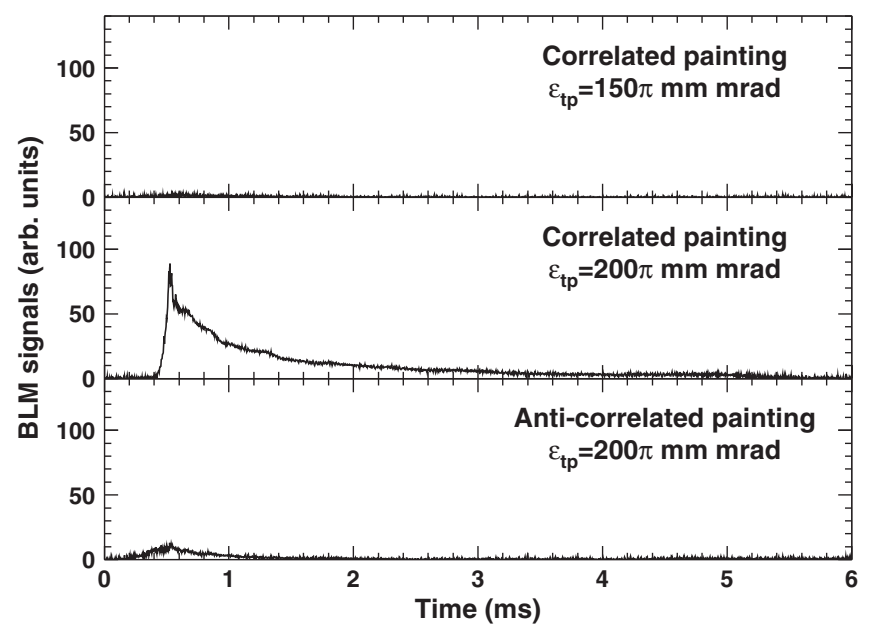

\section{Beam loss mitigation by introducing anticorrelated painting}

Based on the above considerations, we attempted to further expand the transverse painting area from $150 \pi$ to $200 \pi \mathrm{mm}$ mrad. The left plot in Fig. 37 is the experimental result for the beam intensity of $1 \mathrm{MW}$, showing the scintillation-type BLM signals over the first $6 \mathrm{~ms}$ measured at the collimator section with QDTs. The top plot corresponds to the case of correlated painting with $\epsilon_{t p}=150 \pi \mathrm{mm} \mathrm{mrad}$, where no significant extra beam loss is observed thanks to the addition of QDTs. But, for correlated painting of $\epsilon_{t p}=200 \pi \mathrm{mm} \mathrm{mrad}, 1.8 \%$-significant beam loss occurred, as shown in the middle plot. This beam loss is ascribed to additional emittance growth arising from emittance exchange caused by the $2 \nu_{x}-$ $2 \nu_{y}=0$ resonance. As shown in the bottom plot, the beam

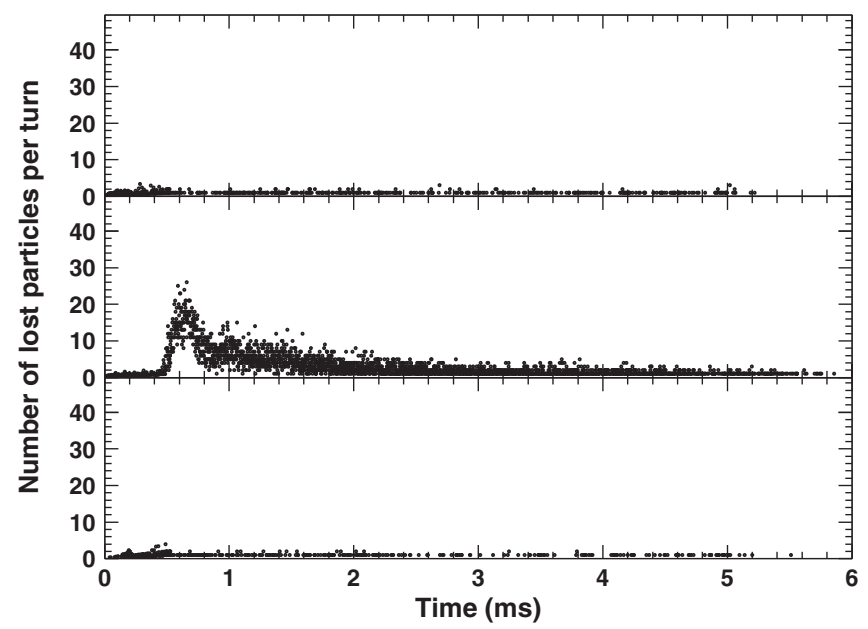

FIG. 37. (Left) Scintillation-type BLM signals over the first $6 \mathrm{~ms}$ measured at the collimator section with QDTs for the beam intensity of $1 \mathrm{MW}$; (top) correlated painting of $\epsilon_{t p}=150 \pi \mathrm{mm} \mathrm{mrad,} \mathrm{(middle)} \mathrm{correlated} \mathrm{painting} \mathrm{of} \epsilon_{t p}=200 \pi \mathrm{mm} \mathrm{mrad,} \mathrm{and} \mathrm{(bottom)}$ anticorrelated painting of $\epsilon_{t p}=200 \pi \mathrm{mm}$ mrad. (Right) Corresponding numerical simulation results. 


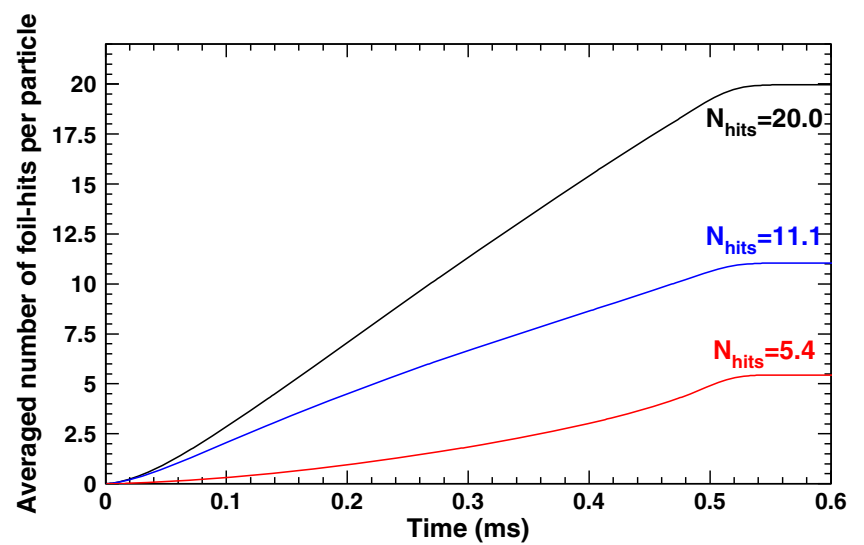

FIG. 38. Averaged number of foil-hits per particle during charge-exchange injection evaluated for correlated painting of $\epsilon_{t p}=100 \pi \mathrm{mm} \mathrm{mrad}$ (black), correlated painting of $\epsilon_{t p}=$ $150 \pi \mathrm{mmmrad}$ (blue), and anticorrelated painting of $\epsilon_{t p}=$ $200 \pi \mathrm{mm} \operatorname{mrad}$ (red).

loss was drastically mitigated by introducing anticorrelated painting, as predicted by the numerical simulation displayed on the right side in Fig. 37.

Although $0.25 \%$-slight extra beam loss, not arising from foil scattering, still remains, as shown in the leftbottom plot in Fig. 37, we expect that it does not lead to serious problems, because its value is small enough; besides most of it can be localized at the collimator section. That is, it is controlled beam loss. The point of this work is to decrease uncontrolled beam loss component. By the above efforts, uncontrolled beam loss arising from large-angle scattering on the charge-exchange foil was drastically reduced. As shown in Fig. 38, the averaged number of foil hits per particle was originally 20.0 with correlated painting of $\epsilon_{t p}=100 \pi \mathrm{mm} \mathrm{mrad}$, but it was finally reduced to 5.4 by the success of anticorrelated painting of $\epsilon_{t p}=200 \pi \mathrm{mm}$ mrad. This reduced number predicts that machine activation near the charge-exchange foil is kept below $10 \mathrm{mSv} / \mathrm{h}$ on the chamber surface, even for the 1-MW beam operation.

Although the above beam test was basically performed by the beam-on-demand single-shot mode, the continuous 1-MW beam operation at $25 \mathrm{~Hz}$ is now estimated to be realized sufficiently within a permissible beam loss level.

\section{SUMMARY}

Following a series of injector linac upgrades in 2013 and 2014, we developed a full-scale high-intensity beam test for establishing a low-loss 1-MW beam operation.

In this work, numerical simulation was successfully utilized along with experimental approaches. Empirical beam losses including their time structures were well reproduced by the numerical simulation within an error of several $10 \%$ or less. The well-established numerical simulation very much helped us in finding beam loss mechanism. In particular, the simulated single-particle behavior of each beam loss particle provided us with decisive information for understanding what was happening behind beam loss. Various practical solutions to mitigate observed beam losses were proposed based on the results of the numerical simulations, and they were validated experimentally. Via such continuous efforts iterating experiments and numerical simulations, and also via several hardware improvements such as the rf power supply upgrade, we have recently established a 1-MW beam operation with considerably low fractional beam loss of a couple of $10^{-3}$.

RCS is now ready to demonstrate the continuous 1-MW beam operation, but it is unfortunate that the routine RCS beam power for users has temporarily been limited to 150 $200 \mathrm{~kW}$ since February 2016 due to a malfunction of the neutron production target at MLF. After the installation of a new neutron production target in 2017, the routine RCS beam power will be increased step-by-step to $1 \mathrm{MW}$.

High-intensity beam studies for RCS will be continued with more careful attention to beam quality as well as beam loss, and also for further beam power ramp-up going beyond $1 \mathrm{MW}$ in the future.

\section{ACKNOWLEDGMENTS}

The authors acknowledge Dr. K. Hasegawa and Prof. T. Koseki, and all the RCS group members for their continuous support and encouragement for the present work. We are also grateful to Dr. S. Meigo for his indispensable support during the beam test, and to Dr. S. Machida for his valuable advice and support for the numerical simulation and its analysis. The authors acknowledge Prof. Y. Yamazaki, Prof. Y. Irie, Prof. E. Takasaki, and Prof. K. Oide for their continuous support and sharp advice for the present work. Finally, we would like to express our special thanks to the late Prof. A. Ando. His rich experience and knowledge on accelerators and also his humor helped us whenever we faced difficulties during beam commissioning.

[1] High-intensity Proton Accelerator Project Team, J-PARC, JAERI Report No. JAERI-Tech 2003-044.

[2] H. Hotchi et al., Beam commissioning of the 3-GeV rapid cycling synchrotron of the Japan Proton Accelerator Research Complex, Phys. Rev. ST Accel. Beams 12, 040402 (2009).

[3] H. Hotchi, H. Harada, N. Hayashi, M. Kinsho, P. K. Saha, Y. Shobuda, F. Tamura, K. Yamamoto, M. Yamamoto, M. Yoshimoto, S. Kato, Y. Irie, T. Koseki, Y. Sato, K. Satou, and M. Shirakata, Beam commissioning and operation of the Japan Proton Accelerator Research Complex 3-GeV rapid cycling synchrotron, Prog. Theor. Exp. Phys. 2012, 02B003 (2012). 
[4] I. Sugai, Y. Takeda, M. Oyaizu, H. Kawakami, Y. Irie, A. Takagi, H. Hattori, and K. Kawasaki, Double-layer effects on the lifetime of newly developed HBC-foils for RCS of J-PARC, Nucl. Instrum. Methods Phys. Res., Sect. A 613, 457 (2010).

[5] K. Yamamoto, Efficiency simulations for the beam collimation system of the Japan Proton Accelerator Research Complex rapid-cycling synchrotron, Phys. Rev. ST Accel. Beams 11, 123501 (2008).

[6] H. Ao and Y. Yamazaki, First high-power model of the annular-ring coupled structure for use in the Japan Proton Accelerator Research Complex linac, Phys. Rev. ST Accel. Beams 15, 011001 (2012).

[7] H. Oguri, K. Ohkoshi, K. Ikegami, A. Takagi, H. Asano, A. Ueno, and T. Shibata, Status of the RF-driven $\mathrm{H}^{-}$ion source for J-PARC linac, Rev. Sci. Instrum. 87, 02B138 (2016).

[8] Y. Kondo, T. Morishita, S. Yamazaki, T. Hori, Y. Sawabe, E. Chishiro, S. Fukuta, K. Hasegawa, K. Hirano, N. Kikuzawa, I. Koizumi, A. Miura, H. Oguri, K. Ohkoshi, F. Sato, S. Shinozaki, and A. Ueno, Beam test of a new radio frequency quadrupole linac for the Japan Proton Accelerator Research Complex, Phys. Rev. ST Accel. Beams 17, 120101 (2014).

[9] S. Machida and M. Ikegami, Simulation of space charge effects in a synchrotron, AIP Conf. Proc. 448, 73 (1998).

[10] L. Schachinger and R. Talman, Teapot: A thin-element accelerator program for optics and tracking, Part. Accel. 22, 35 (1987).

[11] H. Hotchi, F. Noda, Y. Irie, S. Machida, and A. Y. Molodojentsev, in Proceedings of the 2006 European Particle Accelerator Conference (EPAC2006), Edinburgh, Scotland, 2006 (EPS-AG, Edinburgh, Scotland, 2006), p. 2104.

[12] See http://geant4.cern.ch.

[13] H. Hotchi, H. Harada, N. Hayashi, M. Kinsho, P. K. Saha, Y. Shobuda, F. Tamura, K. Yamamoto, M. Yamamoto, M. Yoshimoto, and Y. Irie, Beam loss reduction by injection painting in the $3-\mathrm{GeV}$ rapid cycling synchrotron of the Japan Proton Accelerator Research Complex, Phys. Rev. ST Accel. Beams 15, 040402 (2012).

[14] Y. Kamiya, in Proceedings of the 1989 IEEE Particle Accelerator Conference, Chicago, IL (IEEE, New York, 1989), p. 660.

[15] J. Beebe-Wang, Y. Y. Lee, D. Raparia, J. Wei, C. R. Prior, and S. Machida, in Proceedings of the 2000 European Particle Accelerator Conference, Vienna, Austria, 2000 (EPS, Geneva, 2000), p. 1465.

[16] F. Tamura, M. Yamamoto, M. Yoshii, C. Ohmori, M. Nomura, A. Schnase, M. Toda, H. Suzuki, T. Shimada, K. Hara, and K. Hasegawa, Longitudinal painting with large amplitude second harmonic rf voltages in the rapid cycling synchrotron of the Japan Proton Accelerator Research Complex, Phys. Rev. ST Accel. Beams 12, 041001 (2009).

[17] M. Yamamoto, M. Nomura, A. Schnase, T. Shimada, H. Suzuki, F. Tamura, E. Ezura, K. Hara, K. Hasegawa, C. Ohmori, K. Takata, A. Takagi, M. Toda, and M. Yoshii, Simulation of longitudinal beam manipulation during multi-turn injection in J-PARC RCS, Nucl. Instrum. Methods Phys. Res., Sect. A 621, 15 (2010).
[18] L. J. Laslett, BNL Report No. 7534, p. 325.

[19] Y. Shobuda, Y. Irie, and S. Igarashi, Analytical method for the evaluation of field modulation inside the rf-shielded chamber with a time-dependent dipole magnetic field, Phys. Rev. ST Accel. Beams 12, 032401 (2009).

[20] T. Takayanagi, J. Kamiya, M. Watanabe, Y. Yamazaki, Y. Irie, J. Kishiro, I, Sakai, and T. Kawakubo, Design of the injection bump system of the $3-\mathrm{GeV}$ RCS in J-PARC, IEEE Trans. Appl. Supercond. 16, 1358 (2006).

[21] N. Hayashi, M. Kawase, S. Hatakeyama, S. Hiroki, R. Saeki, H. Takahashi, Y. Terunuma, R. Toyokawa, D. Arakawa, S. Hiramatsu, S. Lee, K. Satou, M. Tejima, and T. Toyama, Beam position monitor system of J-PARC RCS, Nucl. Instrum. Methods Phys. Res., Sect. A 677, 94 (2012).

[22] T. Takayanagi, N. Hayashi, M. Kinsho, T. Ueno, T. Togashi, K. Horino, and Y. Irie, Design and preliminary performance of the new injection shift bump power supply at the J-PARC 3-GeV RCS, IEEE Trans. Appl. Supercond. 24, 0503504 (2014).

[23] K. Okabe, Commissioning of the injection beam for the J-PARC RCS, in Space-charge 2015 workshop, Oxford, UK, 2015 (EuCARD2/XBeams, Oxford, 2015), see https:// www.cockcroft.ac.uk/events/SpaceCharge15/.

[24] F. Tamura, M. Yamamoto, C. Ohmori, A. Schnase, M. Yoshii, M. Nomura, M. Toda, T. Shimada, K. Hara, and K. Hasegawa, Multiharmonic feedforward system for beam loading compensation in wide-band cavities of a rapid cycling synchrotron, Phys. Rev. ST Accel. Beams 14, 051004 (2011).

[25] M. Yamamoto, K. Hara, K. Hasegawa, M. Nomura, C. Ohmori, T. Shimada, F. Tamura, M. Toda, and M. Yoshii, in Proceedings of the 12th Annual Meeting of Particle Accelerator Society of Japan (PASJ2015) (Tsuruga, Japan, 2015), p. 1008.

[26] F. Tamura, H. Hotchi, A. Schnase, M. Yoshii, M. Yamamoto, C. Ohmori, M. Nomura, M. Toda, T. Shimada, K. Hasegawa, and K. Hara, High intensity single bunch operation with heavy periodic transient beam loading in wide band rf cavities, Phys. Rev. ST Accel. Beams 18, 091004 (2015).

[27] Y. Shobuda, Y. H. Chin, P. K. Saha, H. Hotchi, H. Harada, Y. Irie, F. Tamura, N. Tani, T. Toyama, Y. Watanabe, and M. Yamamoto, Theoretical elucidation of space charge effects on the coupled-bunch instability at the $3 \mathrm{GeV}$ rapid cycling synchrotron at the Japan Proton Accelerator Research Complex, Prog. Theor. Exp. Phys. 2017, 013G01 (2017).

[28] P. K. Saha, Y. Shobuda, H. Hotchi, H. Harada, N. Hayashi, M. Kinsho, M. Nomura, F. Tamura, N. Tani, Y. Watanebe, and M. Yamamoto, in Proceedings of the 2016 International Particle Accelerator Conference (IPAC2016), Busan, Korea, 2016 (JACOW, Busan, Korea, 2016), p. 589.

[29] H. Hotchi, N. Tani, Y. Watanabe, H. Harada, S. Kato, K. Okabe, P. K. Saha, F. Tamura, and M. Yoshimoto, Beam loss caused by edge focusing of injection bump magnets and its mitigation in the $3-\mathrm{GeV}$ rapid cycling synchrotron of the Japan Proton Accelerator Research Complex, Phys. Rev. Accel. Beams 19, 010401 (2016). 
[30] H. Hotchi, N. Tani, and Y. Watanabe, Numerical study for beam loss occurring for wide-ranging transverse injection painting and its mitigation scenario in the J-PARC 3-GeV RCS, Nucl. Instrum. Methods Phys. Res., Sect. A 778, 102 (2015).

[31] Y. Wang et al., in Proceedings of the 15th Particle Accelerator Conference (PAC1993), Washington, DC, 1993 (IEEE, New York, 1993), p. 227.
[32] B. W. Montague, CERN-Report No. 68-38, 1968.

[33] A. Ando, T. Suzuki, E. Takasaki, and K. Takayama, Coupling resonance $2 \nu_{h}-2 \nu_{v}=0$ in the KEK proton synchrotron, IEEE Trans. Nucl. Sci. 26, 3541 (1979).

[34] L. M. Kapchinsky and V. V. Vladimirsky, in Proceedings of the International Conference on High Energy Accelerators (CERN, Geneva, 1959), p. 278. 